\title{
Extensive incorporation, polarisation and improved maturation of transplanted human cones in a murine cone degeneration model
}

Authors:

Sylvia J Gasparini*•1, Karen Tessmer*1, Miriam Reh², Stephanie Wieneke ${ }^{1,3}$, Madalena Carido $^{1}$, Manuela Völkner ${ }^{1,3}$, Oliver Borsch ${ }^{1}$, Anka Swiersy ${ }^{1}$, Marta Zuzic ${ }^{1,4}$, Olivier Goureau ${ }^{5}$, Thomas Kurth ${ }^{6}$, Volker Busskamp ${ }^{1,4}$, Günther Zeck ${ }^{2,7}$, Mike O Karl ${ }^{1,3}$, Marius Ader ${ }^{\bullet 1}$

* co-first authors $\bullet$ corresponding authors

Affiliations:

1. Center for Regenerative Therapies Technical University Dresden (CRTD), Dresden, Germany

2. Natural and Medical Sciences Institute at the University of Tübingen, in Reutlingen, Germany

3. German Center for Neurodegenerative Diseases (DZNE) Dresden, Germany

4. University of Bonn, Department of Ophthalmology, Bonn, Germany

5. Sorbonne Université, Institut de la Vision, INSERM, CNRS, 75012 Paris, France

6. Center for Molecular and Cellular Bioengineering (CMCB) of the TU Dresden, Technology Platform

7. Biomedical Electronics and Systems, EMCE Institute, Technische Universität Wien, Austria.

Corresponding author:

Sylvia Gasparini

Marius Ader

Center for Regenerative Therapies Technical University Dresden (CRTD)

Technische Universität Dresden

Fetscherstr. 105

01307 Dresden, Germany

$+49(0) 351-45882203$

sylvia.gasparini@tu-dresden.de

marius.ader@tu-dresden.de

Keywords

Photoreceptor replacement, iPSC, cone reporter, retinal organoid, transplantation, incorporation, outer segment, polarisation 
1

2 Once human photoreceptors die, they do not regenerate, thus photoreceptor transplantation

3 has emerged as a potential treatment approach for blinding diseases. Improvements in

4 transplant organization, donor cell maturation and synaptic connectivity to the host will be

5 critical in advancing this technology to clinical practice. Unlike the unstructured grafts of prior cell suspension transplantations into end-stage degeneration models, we describe extensive incorporation of iPSC retinal organoid-derived human photoreceptors into mice with cone dysfunction. This incorporative phenotype was validated in both cone-only as well as panphotoreceptor transplantations. Rather than forming a glial barrier, Müller cells extend throughout the graft, even forming a common outer limiting membrane. Donor-host interaction appears to promote polarisation as well as development of morphological features critical for light detection, namely formation of inner and well stacked outer segments oriented towards the RPE. Putative synapse formation and graft function is evident both at a structural and electrophysiological level. Overall, these results show that human photoreceptors interact readily with a partially degenerated retina. Moreover, incorporation into the host retina appears to be beneficial to graft maturation, polarisation and function.

- Generation of the first human iPSC cone reporter line

- Human cones extensively incorporate into the retina of mice with cone degeneration

- Donor cone age and time in vivo are important factors for transplant incorporation

- Incorporation into the host retina correlates with graft polarisation 


\section{Introduction}

26

27

Vision impairment represents the most prevalent disability in the industrialized world and very few treatment options exist (1). Many blinding diseases are characterized by the progressive loss of photoreceptor cells which lack the ability to regenerate in mammals, including humans.

Photoreceptor transplantation therapy has thus been proposed as a treatment modality in which healthy donor cells replace those that have been lost. Cell replacement therapies are an attractive option for retinal diseases - the eye is an organ which is self-contained and partially immune privileged, minimizing the risk of unwanted cell migration and rejection (2). Additionally, the eye is readily accessible and easily monitored. The cone rich foveal region, which is extremely important for human vision, facilitating tasks such as reading, facial recognition and driving, is relatively small, reducing the amount of donor cells required. Within the fovea there are only around 200000 cones (3) - a number of cells which is readily produced with current organoid technology. However, to date no human cone-specific reporter line has been described and no efficient cell surface markers have been identified to facilitate effective sorting of donor cones. Although a marker panel for cone enrichment has been suggested, this provided low purity and yield (4).

Several recent studies have utilized human stem cell derived retinal organoids as a source of either photoreceptor or retinal sheets for transplantation, particularly in end stagedegeneration models. While some improvements in vision have been reported through the use of retinal sheets (5-9), these grafted sheets were largely disorganized, with extensive rosette formation and the complication of donor photoreceptors mostly synapsing to donor second order neurons rather than host cells. For human cone cell suspension transplantations, 
while functionality has recently been reported from two different research groups, grafts appeared disordered with little evidence of cell polarisation (10) (i.e. inner and outer segments oriented towards RPE, axons extended towards the second order neurons) or showed some polarisation but poor general transplant cell survival $(11,12)$.

The aforementioned studies mostly focused on transplantation into models of severe endstage degeneration, particularly the $\mathrm{rd} 1$ mouse model, where no host photoreceptors remained. While these proof-of-concept studies are of utmost importance for the development of photoreceptor replacement therapies, the early onset and severity of the rd1 phenotype represents a rather atypical pathology in regard to retinal degeneration patients. Complete photoreceptor cell loss only occurs in patients in very late stages of retinal disease, while in AMD, the most prevalent retinal degenerative disease, massive photoreceptor degeneration, called geographic atrophy (GA), is locally restricted. Additionally, a highly degenerated environment may not be conducive to graft survival, organized graft integration or synaptic connectivity with the host retina. In humans, extensive glial scarring and neural retinal remodeling may render end stage transplantations challenging (13). It is not yet known in which retinal disease type or at what degenerative stage photoreceptor replacement therapies would be most effective. Here, we therefore used the cone photoreceptor function loss mouse (Cpfl1), in which cones are dysfunctional and rapidly degenerate while rods remain largely unaffected (14), in order to determine whether human cones can integrate into the existing host photoreceptor layer.

In this study, a cone-specific human iPSC GFP-reporter line was generated in order to facilitate FAC sorting of an enriched cone population from retinal organoids. We used an optimized 
73 differentiation protocol which generates cone-rich retinal organoids ensuring a large

74 population of transplantable cone cells. We aimed to investigate how transplanted cones

75 mature, as well as how the donor-host interaction changes over time after transplantation.

76 Results show long-term survival for up to six months in mouse recipients, extensive and

77 polarised incorporation into the remaining mouse outer nuclear (photoreceptor) layer and

78 interaction with host Müller glia and second order neurons. Human graft incorporation was

79 further validated through the use of donor photoreceptors from a pan-photoreceptor

reporter iPSC line. Moreover, photoreceptor graft maturation and polarisation was enhanced

by donor-host interaction, as shown by histology, ultrastructural analysis and transcriptomics.

Human photoreceptor transplants ultimately led to the re-establishment of cone-mediated light-responses in the cone deficient mouse.

A human iPSC line carrying GFP under the control of the cone-specific mouse cone arrestin (mCar) promoter was generated using a piggyBac transposon system (mCar-GFP line). This did not affect karyotype (Fig S1B,C). Human mCar-GFP retinal organoids were produced using a modified version of a previously published protocol which has been shown to generate robust numbers of cone photoreceptors (Fig S1A)(15-17). The mCar-driven GFP signal was predominantly located in the outer neuroepithelial layer as to be expected for cones (Fig 1A). 
reporter. The $\mathrm{GFP}^{+}$cells were positive for the photoreceptor-specific markers CRX and recoverin, and also expressed more mature cone markers such as L/M Opsin, and S-opsin at day (D) 240 of in vitro differentiation (Fig 1B). Note that there are far more L/M opsin cones present in the organoids than S-opsin cones, as previously described (15). Markers of other retinal cell types, namely, rods (Nrl and Rhodopsin), Müller glia (Sox2 and GLAST/CRALBP), bipolar (PKC $\alpha$ ) and amacrine/ganglion cells (HuC/D) did not colocalize with GFP (Fig S1D-F). For a more in-depth analysis of the cell identity of GFP expressing cells, next generation sequencing of FAC-sorted $\mathrm{GFP}^{+}$and $\mathrm{GFP}^{-}$cells was performed with D200, D270 and D370 retinal organoids. This analysis confirmed that $\mathrm{GFP}^{+}$cells highly express cone specific genes such as ARR3, CNGB3, PDE6C and L and $M$ opsins, whereas the negative fraction showed high expression of typical marker genes of other retinal cell-types including rods, Müller glia, bipolar cells and retinal ganglion cells (Fig 1B). Additionally, Gene ontology term analysis of differentially expressed genes in cones from D200 versus D270 organoids revealed an enrichment of cellular compartment pathways critical to photoreceptor function in D270 cones, indicating that D200 cones are not yet fully mature and undergo extensive molecular changes in the following 10 weeks (Fig 1C).

To assess the proportion of cones in the organoids and the efficiency of reporter expression, FAC-sorting followed by immunocytochemical analysis was performed (Fig 1D-G). As expected, there was a significant increase in the proportion of $\mathrm{GFP}^{+}$cells with organoid age (i.e. at D140, D200, D250), with up to $45 \%$ of cells determined to be $\mathrm{GFP}^{+}$by D250 (Fig 1D). The FAC-sorted GFP ${ }^{+}$fraction was found to be highly enriched in recoverin and ARR3 positive cells (Fig 1E-G), whereas the GFP- fraction was almost entirely depleted of cone arrestin positive cells at all time points investigated (Fig 1G). This indicates that almost all cones are 
121 captured using the mCar-GFP reporter-based sorting system. With the confirmation of the

122 cone identity of $\mathrm{GFP}^{+}$cells, cones from D200 organoids were determined to be the most

123 suitable population to perform transplantation studies, due to the robust number of relatively

124 mature cone cells present, combined with a high degree of viability following dissociation and

125 FACS purification. A smaller transplantation study using cones from D250 organoids was also

126 performed for comparison.

127

Human cones incorporate extensively into the host retina with longer post-transplantation

\section{9 times}

131 Human cones were transplanted into the subretinal space (between the RPE and

132 photoreceptor layer) of Cpfl1 mice, which received monthly vitreal triamcinolone acetonide

133 injections for immune suppression from the time of transplantation. All transplanted cells expressed human ARR3 across the study timeframe and minimal immune reactivity of the host

in the subretinal space but did not interact extensively with the host outer nuclear layer (ONL).

137 Donor cell clusters appeared mostly separated from the host ONL with few contact points (Fig 
generation sequencing revealed the human origin of $\mathrm{GFP}^{+}$cells isolated from transplanted

146 retinas (see below).

In addition to incorporating into the host ONL over time, human cones also appear to further

157 rich bulbous outgrowths are indicative of inner segment development (Fig $2 A, B$ ). These

2C). To confirm the inner segment identity of the bulbous mitochondria-rich outgrowths, retinal sections were stained with markers associated with inner and outer segments.

161 Accordingly, PNA which is specific for cone inner and outer segments, was bound in a non-

162 localised fashion throughout the graft at 3 weeks. By 10 and even more prominently by 26

163 weeks, the PNA label was increasingly concentrated in mitochondria rich regions, i.e. the RPE

164 facing edge of incorporated grafts and the rosette-like structures which occurred in some 165 areas where mouse photoreceptors remained underneath the incorporating graft (Fig 3A). 166 Peripherin-2 (PRPH2) staining of outer segments was not evident in the human cones at 3 
was expressed in close association with the putative inner segments (hMito), also suggesting

170 To investigate the extent of photoreceptor maturation further, grafts were examined at the

171 ultrastructural level. Indeed, many examples of inner segments were seen at 10 weeks post-

172 transplantation, whereas outer segments were not found (Fig 3C). By 26 weeks, however,

173 numerous cones formed relatively well organized and tightly stacked outer segment-like

174 structures which were sometimes found to be joined to inner segments via a connecting

175 cilium, additionally identifiable by the characteristic basal bodies (Fig 3D,E). The cells

176 displaying these photoreceptor specific features were confirmed to be of human origin by the

177 distinctive size and morphology of the human cone nuclei (these are much larger and less

178 electron dense than the mouse photoreceptor cells - e.g. Fig 5A,B) as well as through

179 immunogold labelling of human specific ARR3 (Fig S2C).

181 As inner and particularly outer segments took a long time to develop post-transplantation, we

182 postulated that transplanting cones derived from older organoids might reduce the time

183 required for the in vivo development of such mature photoreceptor specific features. Cones

184 isolated from D250 retinal organoids were transplanted and assessed 10 weeks post-

185 transplantation. Interestingly, unlike D200 cones, after 10 weeks in vivo most of the D250

186 grafts remained in the subretinal space, indicating a reduced capacity of the older cells to

187 incorporate into the host ONL (Fig 3F). Much like D200 + 3 week transplantations, the

188 D250 + 10 week grafts were presenting as a largely amorphous cell mass with few

189 mitochondria rich or PRPH2 outgrowths evident and PNA label dispersed through the cell

190 mass, rather than accumulating towards the RPE (Fig $3 F, G, H)$. At an ultrastructural level,

191 occasional inner segments as well as some outer segments were observed, however, the outer 
192

193

194

195

196

197

198

199

200

201

202

203

204

205

206

207

208

209

210

211

212

213

214

segments were highly disorganized and not tightly stacked (Fig 3I,J). Although photoreceptors

of D250 + 10 week grafts (i.e. post-differentiation D320) are in total older than D200 +

10 week grafts (post-differentiation D270), they in comparison show a decreased capacity for

incorporation and maturation. This suggests D200 cones are a preferable donor cell age.

Together, these observations indicate that donor cone age and time in vivo are important

factors for transplant incorporation and maturation.

\section{Müller glia incorporate transplanted cones into the host retina, forming a common OLM}

In normal retinal physiology, photoreceptors are intermingled in a dense network of Müller glia processes that support photoreceptor structure, homeostasis, and function - even participating in the cone visual cycle (22). Therefore, the interaction between transplanted human cones and recipient Müller glia was assessed.

Immunohistochemical staining for GFAP revealed that in the D200 + 3 week and D250 + 10

week transplants, Müller glia processes extend into the graft only in limited areas where donor clusters start to make contact with the ONL, while no GFAP staining was observed within subretinal-located grafts (Fig 4A). By D200 + 10 weeks, rather than forming a glial barrier, Müller glia processes permeate throughout the graft (Fig 4A), and seemingly create an outer limiting membrane (OLM) in between the human nuclei and the subretinal space. Phalloidin staining supports this finding, showing an actin-dense band above the human nuclei which is continuous and in line with the host OLM, incorporating the clusters of human cones rather than excluding the xenogeneic cells (Fig 4B). This interaction was maintained at 26 weeks (Fig $4 A, B, D)$. 

between human cones and endogenous photoreceptors (Fig 4C).

221 clusters of incorporated human cones that mature photoreceptor-specific features of inner largely amorphous (Fig 5A,B).

To quantify the extent of donor-host interaction at different experimental timepoints, total

calculated. Here, "ONL contact" was defined as areas where the cell mass remains in the sub-

was defined as areas where the transplant was in line with the host ONL (Fig 5C), but where

235 incorporated and a further $20 \%$ fully incorporated into the host ONL. By D200 + 26 weeks,

236 over $40 \%$ of the graft area was fully incorporated. Both the D200 + 3 week and the D250 + 10

237 week samples only minimally interacted with the host retina ( $85 \%$ graft area non-interacting)

238 (Fig 5D). Accordingly, only D200 + 10 week and D200 + 26 weeks grafts exhibited numerous 
239 mitochondrial rich outgrowths, i.e. inner segments (Fig 5E). If this were simply a factor of cell

240 age, one would expect D250 + 10 week to display at least as many inner segments as D200 +

24110 week grafts, however, in line with our previous observations, these only developed very

242 few mitochondria-rich inner segments. Moreover, where inner segments did develop, these

243 appear almost exclusively in areas where the host retina is directly contacted by the graft (Fig

$2443 F)$, indicating that interaction with the host influences the maturation and development of

245 photoreceptor-specific morphological features like inner segments.

Cones mature more extensively in the mouse retinal environment compared to those

248 maintained in retinal organoids in vitro

250 In order to further investigate whether the maturation trajectory of the retinal organoid-

251 derived cones was influenced, as we suggest, by the host retinal environment, we compared

252 the transcriptional profile of transplanted cones with cones from age-matched retinal

253 organoids. D200 organoids were either maintained for a further 10 or 26 weeks (henceforth

254 referred to as in vitro) or whole eye cups were dissociated at 10 - and 26-weeks post 255 transplantation (hereafter referred to as in vivo), and $\mathrm{GFP}^{+}$cells recollected via FACS for RNA 256 sequencing (Fig 6A). Interestingly, PCA analysis of the top 500 differentially regulated genes 257 revealed that the greatest source of variance in the data separated clusters not depending on 258 their age (D200 + 10 week and D200 + 26 week in vitro samples cluster closely together in 259 PC1), but according to the time in vivo, indicating that maturation within the host retina 260 indeed plays an important role (Fig 6B). More detailed gene overrepresentation analysis 261 showed that molecular mechanisms, biological processes and cellular compartment pathways 262 involved in light perception were highly and significantly enriched in the in vivo matured cone 

formation, which is critical to light detection.

To examine whether the incorporating capacity displayed by the human cones was specific to this cell line, we generated and transplanted photoreceptors from a CRX driven mCherry reporter iPSC line (23). CRX is expressed in retinal progenitors, rods and cones, with CrxmCherry thus marking both photoreceptor cell types (Fig S3). FAC-sorted D200 Crx-mCherry ${ }^{+}$ cells were transplanted into Cpfl1 mice as per mCar-GFP ${ }^{+}$cones. A remarkably similar whole sections of mouse ONL (Fig 7A,B), with apical oriented inner segments (Fig 7A-D) and

Müller glia extensions throughout the graft (Fig 7E). 
showed that both $\mathrm{PKC}^{+}$rod- and segretagogin ${ }^{+}$cone- bipolar cell dendrites extended extensively into human cone clusters in areas where the donor cells are incorporated into the host ONL (Fig 8A; S3A). Further, calbindin ${ }^{+}$horizontal cells also extended neural processes into the human cone grafts (Fig S3B). These observations indicate potential synaptic connections formed between donor cones and host second order neurons. To further investigate connectivity between donor and host cells, association between pre- and post-synaptic markers was assessed. As seen in Fig 8B, many examples of ribbon synapses labelled by CTBP2 within the graft can be found in close proximity to the bipolar cell post-synaptic marker mGluR6. This further supports putative synaptic connectivity between graft and host. Finally, the presence of typical photoreceptor ribbon synapses was confirmed by EM already at 10 weeks post-transplantation (Fig 8C).

To evaluate the functionality of these potential connections, we performed 300 electrophysiological measurements using multi-electrode array (MEA) recordings. Here, due 301 to technical challenges associated with cell mass localization of GFP causing severe bleaching, 302 retinas containing Crx-mCherry cells were used. Robust and stable ON and OFF photopic light 303 evoked responses (30 minutes of binary checkerboard white noise stimulation with stringent spike threshold settings to reduce artifacts) were detected in 5 of 9 transplanted eyes tested 305 (Fig 9 A, B, E, F, G). However, low levels of photopic light responsiveness were also detected in non-transplanted regions of the same retina (Fig $9 \mathrm{E}, \mathrm{F}$ ), but only following fluorescent stimulation, which was necessarily applied to locate the cell mass. Rods have been reported to respond to photopic light when over saturated (24). To eliminate potential endogenous 309 oversaturated rod activity, the metabotropic glutamate receptor blocker L-AP4 was added 310 during recording. L-AP4 blocks synaptic transmission between photoreceptors and all ON 
bipolar cells, including rod bipolar cells. Spike-triggered averaging was then used to categorise

312 the ganglion cell response types (Fig 9 B, D). As expected, L-AP4 effectively quenched all ON

313 RGC responses (Fig 9E, H). Moreover, OFF responses which are driven by cone bipolars

314 remained only in the transplanted region (Fig 9F), strong evidence that the light-induced

315 spiking activity is driven by the transplanted photoreceptors due to the lack of functional

316 endogenous cones. Note that when the receptive field of the active ganglion cells was

317 calculated, there was a high degree of overlap with the cell mass location (Fig 9C), further 318 indicating that the transplant is driving the functional response to photopic light.

In this study a human cone-specific GFP-reporter iPSC line facilitated the efficient enrichment of human cone photoreceptors from retinal organoids. The use of a local immune suppressant, monthly vitreal injection of triamcinolone acetonide, prevented the rejection of 326 these human cells when transplanted into the Cpfl1 mouse subretinal space. This allowed 327 long-term follow up over a 6-month period (26 weeks). With longer transplantation times, grafts interacted extensively with the host retina. These findings were confirmed through 329 transplantations of a second photoreceptor-specific reporter iPSC line. Rather than forming a 330 glial barrier, Müller glia intermingled throughout the graft, leading to the establishment of a 331 common OLM between mouse and human cells. Second order neurons extended dendrites into the transplant forming potential synaptic connections. The incorporation of transplanted

333 human cones into the host retina was accompanied by an improvement in cell polarisation 334 and maturation of photoreceptor-specific morphological features, namely inner and outer 
segments. Light detecting capacity and putative synaptic connectivity of transplanted human downstream retinal ganglion cells.

While human photoreceptor and rod specific ESC/iPSC reporter lines have been previously generated $(23,25-28)$, no human cone reporter PSC line has thus far been described. Based on immunohistochemical and transcriptional assessment, the herein presented mCar-GFP iPSC reporter line appears to robustly and specifically label human cone photoreceptors. This is not only useful for transplantation studies, but may also be of interest in other applications,

e.g. studying human cone development or in the identification of human cone-specific cell surface markers. A previous study used viral labelling of $L / M$ opsin cones to allow 346 identification of cone cell surface markers. Not only does this exclude S-cones, but also, due 347 to viral transduction efficiency, only around half of the total cone population was labelled (4).

348 The resulting marker panel led to a maximal enrichment of $\sim 50 \%$ cones. A pan-cone reporter 349 line would be of use in this context, as identification of cell surface markers is highly 350 advantageous in a clinical setting where reporter or virally labelled fluorescent cells cannot be 351 used.

353 In this study we show extensive incorporation of human cones and Crx-mCherry ${ }^{+}$ 354 photoreceptors into the mouse ONL. This is to our knowledge the first report of such extensive 355 incorporation of donor photoreceptors into the host retina from any species. Mouse into 356 mouse photoreceptor transplantations largely result in material transfer rather than 357 structural integration (18-20) - a mechanism that was ruled out in this study. As most recent 358 studies of human photoreceptor suspension transplantation were either performed over a 
shorter time period and/or focused on transplantation into a fully degenerated retina (10-12,

incorporation due to insufficient time (at 3 weeks only limited interaction was seen) or lack of

stretches of host photoreceptors with no obvious physical impediment to the host INL.

374 Single cell suspension studies have often been criticized for the lack of structure of the 375 resulting graft (30). While in theory retinal sheet transplantation could provide pre376 established structure, currently described studies suffer from extensive rosette formation and 377 self-synapsing to graft second order neurons $(5,7-9,31,32)$. Sheet transplantations are 378 surgically more challenging, particularly in the context of degenerative retinas where rupture 379 of the tissue remains a potential risk. In this study, however, pre-purification of the 380 transplanted cells was possible due to our photoreceptor-specific reporter lines and the used 381 suspension technique. Unlike in other studies, the incorporated cones and Crx-mCherry ${ }^{+}$ 382 photoreceptors appeared to become well polarized, with axon projections towards the INL 
and inner and outer segments towards the RPE. As photoreceptor loss is not complete until

In the present work, graft maturation capacity was only observed upon incorporation into the

host ONL. Through recovery of transplanted cells for next generation sequencing - a

in vivo matured cones from timepoints with extensive incorporation exhibit significant upregulation of visual transduction and outer segment related genes. With longer post- 
While several studies have shown evidence of nascent outer segment formation - often in the with limited and disorganised discs $(21,37-41)$. A recent exception to this is the small but (10). The outer segments seen in our study (D200 + 26 weeks) were not only tightly stacked and relatively well organized but were also seen sometimes to project from the inner segment via a connecting cilium, a feature which, to our knowledge, has not previously been reported in human photoreceptor suspension transplantations. Of note, organised outer segment

414 formation including connecting cilium has been described in retinal sheet transplants (32), 415 however these formed primarily within rosettes which would likely negatively affect function.

416 A recent paper transplanted optogenetically engineered photoreceptors to circumvent the 417 necessity for OS formation (42). While restored visual function was observed, this is limited to 418 the specific wavelength of the optogene and has different kinetics to normal visual perception. 419 Greater understanding and ideally modification of the factors required to encourage 420 transplanted photoreceptors to develop and correctly form distinctive OS structures critical

421 for light detection is of great importance if photoreceptor cell replacement therapy is to be 422 an effective treatment modality.

424 Further interaction of host and donor tissue was seen at the level of the second order neurons.

425 Rod and cone bipolar cells as well as horizontal cells extended dendrites into the transplant.

426 Close proximity of pre- and postsynaptic ribbon synapse proteins supports the putative 427 formation of synaptic connections. Of note, the putative synaptic connectivity occurred 428 already at 10 weeks, preceding the extensive maturation of donor cells, as is also seen during 429 development. Similar plasticity in second order neurons was already described in rd1 mice 
upon photoreceptor transplantation (10), but it is interesting that this effect is also seen in

431 the Cpfl1 host where rod photoreceptors largely remain. This implies that the incorporated

432 cell mass can replace existing connections, as dendritic remodeling of host second order

433 neurons was observed only in areas of human cone incorporation. Similarly, in the

434 aforementioned study, photopic light evoked responses by MEA were also reported (10). In

435 our context, MEA recordings were complicated by the oversaturation of endogenous rods due

436 to fluorescent cell mass localization, leading to a low level of background photopic response.

437 For future studies, the injected cell number may be increased to expand graft area removing

438 the need to locate by fluorescence, as per Ribeiro et al, where the transplantation of 500,000

439 donor cells not only increased graft area but also improved maturation compared to their

440 previous studies using 150,000 cells (10). Regardless, using just 150,000 donor

441 photoreceptors, we observed 3 to 4-fold higher proportion of both ON and OFF responsive

442 RGCs under mesopic and photopic conditions when comparing regions containing

443 transplanted cells with non-transplant containing regions. This is a strong indicator that the

444 increased response is due to light-evoked responses transmitted from the graft. While the ON

445 RGC contribution of the graft vs endogenous rods cannot be resolved definitively, the

446 introduction of L-AP4 isolates cone OFF bipolar responses. As cones are dysfunctional or

447 absent in the Cpfl1 host, any cone-OFF bipolar response should be due to newly formed

448 connections to the graft. Indeed, all ON responses were quenched by L-AP4 and OFF responses

449 remained only in the transplanted region, giving strong evidence that there is photopic light

450 evoked signal transduction of transplanted cells through the cone OFF pathway. This indicates

451 that the well matured and structurally incorporated photoreceptors in this study are

452 functionally integrated and synaptically connected to the host retina. 
In this study we describe the first human cone-specific reporter iPSC-line for the use of retinal

456 incorporated into a mouse model of cone degeneration. Incorporated grafts were well

457 polarized and developed inner and outer segments. Further studies will be required to

458 investigate details of the cellular and molecular requirements for structural incorporation and

459 interaction with the host tissue allowing subsequent donor photoreceptor maturation. Such

460 knowledge will be helpful to further optimize graft organization, OS formation and synaptic

461

Prabhakara Shastry, Klara Schmidtke and Lynn Ebner.

ReSight - 01EK1613A to

to M.A., 01EK1613E to

M.R. and

G.Z)., and

Deutsche

Forschungsgemeinschaft (DFG): within the SPP2127 Program (AD375/7-1 to M.A.), and FZT 
479 ReSight (01EK1613A); DFG KA2794/5-1 SPP2127 (M.K.). This work received financial support

480 from the State Ministry of Baden-Wuerttemberg for Economic Affairs, Labour and Tourism

481 (M.R. and G.Z).V.B. acknowledges funding by the European Research Council (ERC-2020-PoC-

482966709 - iPhotoreceptors), by the Deutsche Forschungsgemeinschaft (SPP2127, EXC-2151-

483390873048 - Cluster of Excellence - ImmunoSensation2 at the University of Bonn) and the

484 Volkswagen Foundation (Freigeist - A110720).T.K. and the EMF of the CMCB are supported by 485 EFRE.

486

487

\section{Author contribution}

488

489

S.G., K.T., and M.A. conceived this study. S.G., K.T., M.R., M.C., O.B., S.W., A.K., M.Z., M.V.,

490

T.K., O.G., M.K., V.B., G.Z. and M.A. designed and/or performed the experiments. S.G., K.T.

491 and M.A. wrote this paper with input from all authors.

492

493

Declaration of interests

494

495 The authors have no disclosures.

496 
497

498

499

500

501

502

503

504

505

506

Methods

\section{Vector production}

The piggyBac vector backbone PB-TRE-dCas9-VPR (43) was a gift from George Church (Addgene plasmid, 63800). All promoter elements and open reading frames between the core insulator at the $5^{\prime}$ and the SV40 polA at the $3^{\prime}$ ends were removed using restriction enzymes and replaced with either PCR-amplified rod or cone reporter cassette. PCR products were introduced into the piggyBac vector backbone using Gibson assembly cloning (44). For the cone reporter cassette production, a PCR-amplified mouse cone arrestin promoter (mCAR) from LV-mCAR-eNpHR-EYFP (45) (gift from Botond Roska) was assembled with an EGFP followed by a downstream WPRE-BGH-pA element. Finally, a PCR-amplified ubiquitin C promoter (UBC)-blasticidin (Bla) cassette from vector pLV-TRET-hNgn1-UBC-Bla (46)(gift from Ron Weiss, Addgene plasmid, 61473) was further added to both vector assemblies resulting in reporter plasmids PB-hRHO-DsRed-WPRE-BGH-pA-UBC-Bla and PB-mCAR-EGFP-WPREBGH-pA-UBC-Bla. The plasmid DNA was transformed in chemically competent bacteria (One Shot $^{\circledR}$ Stbl $3^{\mathrm{TM}}$, Thermo Fisher Scientific) following the manufacturer's protocol. The correct sequences were confirmed with Sanger sequencing. While RFP was also introduced under the Rhodopsin promoter, almost no RFP signal was detected even after 270 days in culture, however for the purposes of a cone transplantation study this was deemed irrelevant (data not shown).

Generation of a hiPSC cone reporter line

519 The Personal Genome Project hiPS cell line PGP1 (47) was a gift from George Church 520 (https://www.encodeproject.org, accession number: ENCBS368AAA). The cells were cultured 


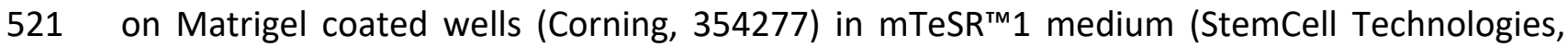

52285850 ) and passaged in the presence of ROCK Inhibitor InSolution ${ }^{\mathrm{TM}}$ Y-27632 (Merck Millipore,

523 688001). The 4D-Nucleofector ${ }^{\mathrm{TM}}$ System (Lonza) was used to electroporate piggyBac and

524 transposase vectors into PGP1 cells in suspension (X-Unit, P3 Primary Cell 4D-Nucleofector ${ }^{\circledR} \mathrm{X}$

525 Kit L, program CB-156) following the manufacturer's protocol. After nucleofections, cells were

526 selected with $20 \mu \mathrm{g} / \mathrm{ml}$ Bla (Thermo Fisher Scientific, A1113903) for five days. The selected

527 cells were seeded at low densities and propagated until each single cell formed a colony.

528 Colonies were picked and genotyped using primers specifically binding to rod and cone

529 reporter cassettes. The monoclonal cell line carrying both reporter cassettes (PGP1dR) at

530 passage number 33-38 was used for all further experiments.

531 hRHO_for - GGATACGGGGAAAAGGCCTCCACGGCCACTAGTAGTTAATGATTAACCCG

532 hRHO_rev - GACGTCCTCGGAGGAGGCCATGGTGGCTGCAGAATTCAGGGGATGACTCT

533 mCAR_for

534 CTGGGGGGATACGGGGAAAAGGCCTCCACGGCCACTAGTGGTTCTTCCCATTTTGGCTAC

535 mCAR_rev

536 GAACAGCTCCTCGCCCTTGCTCACCATGGTGGCTCTAGACCTCCAGCTCTGGTTGCTAAGCTGGC

537

538 hiPSC maintenance and differentiation of retinal organoids

539 The mCar-GFP and Crx-mCherry iPSC lines (kind gift from Olivier Goureau - see (23)) were

540 maintained in mTeSR1 (Stem cell technologies) on matrigel coated plates and split using

541 ReleSR at room temperature (Stem cell technologies). Stem cells were differentiated to retinal

542 organoids using an optimized protocol as previously described see also supplementary

543 methods (17). 


\section{FAC-sorting of reporter positive cells}

545 Retinal organoids were dissociated in $20 \mathrm{U} / \mathrm{ml}$ papain, followed by gentle titration with a fire 546 polished glass pipette and further processing as per the manufacturer's instructions - Papain

547 Dissociation System (Worthington). The cell pellet was resuspended in MACS buffer $(0.5 \%$ 548 BSA, 2 mM EDTA in PBS) to a concentration of $\sim 5$ million cells per $\mathrm{mL}$. The cell suspension was 549 filtered through a $35 \mu \mathrm{m}$ mesh and kept on ice for FAC sorting. An Ariall or Arialll sorter was 550 used to sort GFP ${ }^{+}$or mCherry ${ }^{+}$cells. Briefly, forward (FSC-A) and side scatter area (SSC-A) was 551 used to discriminate cells from debris. Doublets were removed by gating FSC area vs height 552 and by SSC height vs width. Dead cells were gated out using DAPI staining. Finally, GFP ${ }^{+}$or 553 mCherry ${ }^{+}$cells were discriminated from auto fluorescent cells using GFP vs PE or APC.

Adult cone photoreceptor function loss 1 (Cpfl1) mutant (7-14 week-old) were used as recipients for cell transplantation. Mice were maintained in a 12-hour Light/Dark cycle with ad libitum access to food and water.

Following FAC sorting, $\mathrm{GFP}^{+}$or $\mathrm{mCherry}^{+}$cells were resuspended in MACS buffer (150 000 cells $/ \mu \mathrm{l}$ ) and injected into the subretinal space of host eyes as previously described -see also supplementary (48). Directly following cell transplantation, $1 \mu \mathrm{L}$ of preservative free triamcinolone acetonide suspension $(80 \mu \mathrm{g} / \mu \mathrm{L}$ in $\mathrm{NaCl}$ prepared by University Clinic Pharmacy, Dresden) was injected into the vitreous using a hand held $10 \mu$ l Hamilton syringe 
with a blunt 34-Gauge needle. Triamcinolone vitreal injections were repeated on a monthly

569 basis.

570

571 Immunohistochemistry

572

573 Immunohistochemistry was performed as described previously (18) see supplementary

574 methods for details. For immunocytochemistry following dissociation and sorting of the

575 retinal organoid cells, cells were resuspended in RM2 media and laminin was added to each

576 fraction. From each fraction 50000 cells were plated into flexiperm wells on a PDL coated

577 slide. Cells were incubated at $37^{\circ} \mathrm{C}$ for 2 hours to allow attachment. Cells were then fixed for

57815 minutes at room temperature (RT), washed 3 times with PBS and stained as per frozen

579 sections above. Frozen sections and plated cells were mounted following antibody staining

580 using AquaPolymount (Polysciences, Heidelberg, Germany) and imaged using a Zeiss Apotome

581 ImagerZ2 (Zeiss, Heidelberg, Germany).

582

583

Transmission Electron Microscopy (TEM) and Correlative Light Electron Microscopy (CLEM)

584

585 TEM of transplanted cones was performed as previously described $(49,50)$.

586 CLEM of immunolabeled sections was performed as described previously $(51,52)$

587 TEM imaging was performed with a Jeol JEM1400 Plus transmission electron microscope

588 (camera: Ruby, Jeol) running at $80 \mathrm{kV}$ acceleration voltage. 
591

592 Whole eye cups of transplanted eyes or organoids maintained in culture from the same

593 differentiation round were dissociated with papain as described above (Papain dissociation

594 kit 20U). Cell were resuspended in MACS buffer and filtered through a $35 \mu \mathrm{m}$ mesh before 595 FAC-sorting and sequencing - method was modified based on (53) see supplementary for 596 details.

597

598

599

Transcriptomic Analysis

600

601 Basic quality control of the resulting sequence data was done with FastQC (v0.11.6)

602 (https://www.bioinformatics.babraham.ac.uk/projects/fastqc/) and the degree of mouse 603 contamination

was

assessed

with FastQ-Screen

604 (https://www.bioinformatics.babraham.ac.uk/projects/fastq screen). Reads originating from 605 mouse were removed with xengsort (v2021-05-27)(54). Reads were aligned to the human 606 reference genome hg38 using the aligner gsnap (v2020-12-16)(55) with Ensembl 92 human 607 splice sites as support. Uniquely mapped reads were compared based on their overlap to 608 Ensembl 92 human gene annotations using featureCounts (v2.0.1)(56) to create a table of 609 fragments per human gene and sample. Normalization of raw fragments based on library size 610 and testing for differential expression between the different cell types/treatments was 611 performed with the R package DESeq2 (v1.30.1) (57). Sample to sample Euclidean distance, 612 Pearson' and Spearman correlation coefficient and principal component analysis based upon 613 the top 500 genes with the highest variance were computed to explore correlation between 
620 discovery rate (padj $\leq 0.05$ ) were considered as significantly differentially expressed.

A Glass MEA with 256 electrodes of $30 \mu \mathrm{m}$ diameter and a spacing of $200 \mu \mathrm{m}$ spanning an area

The preparation of ex vivo retina was performed in carbonated $\left(95 \% \mathrm{O}_{2}, 5 \% \mathrm{CO}_{2}\right)$ Ames' eyes were opened via a small needle incision above the ora serrata. After removal of the lens,

631 the eye was cut in half and the graft located with stereomicroscope (Leica M80), equipped 632 with a fluorescent illumination unit. The retina with the graft was then separated from the 633 sclera and RPE, trimmed with a scalpel and the vitreous removed. The retina was placed 634 ganglion cell side up on a filter paper and transferred retinal ganglion cell (RGC) side down on 635 to the coated (Cell-Tak, Corning), as described in detail in a previous report (60) recording 636 electrodes and filter paper removed. The other half of the retina was prepared in the same 637 way as a reference sample. 
639 A patterned light stimulus created by an oLED display (DSVGA monochrome green XLT, 640 eMagin) in combination with the software GEARS (61) was used, allowing for binary 641 checkerboard white noise (bwn) stimulation. The oLED is coupled to the microscope with an 642 adapter and its light is projected onto the sample though a $2.5 x$ objective. The oLEDs power 643 was derived as $\mathrm{P}=0.7 \mu \mathrm{W}$ for full-field illumination, which can be calculated into 644 photoisomerizations equaling to approx. $1 \cdot 10^{5} \mathrm{R} /$ photoreceptor/s for both rods and $\mathrm{m}$ cones. We generated pseudo-random, binary (green and black) checkerboard stimuli - where at every stimulus frame the intensity of each checker was drawn from a binary distribution - with temporal frequency of $38 \mathrm{~Hz}$ (frame duration of $26 \mathrm{~ms}$ ) and a total duration of $25 \mathrm{~min}$, with a resolution of 30 pixel x 30 pixel, resulting in an illuminated area of $3.2 \mathrm{~mm} \times 4.2 \mathrm{~mm}$.

During RGC activity recording the MEA chamber was continuously perfused with Ames' solution at a rate of $2-4 \mathrm{~mL} / \mathrm{min}$. The temperature of the MEA chamber was maintained at voltages were recorded using the software MCRack (MCS) and preprocessed using a 2nd order Butterworth highpass filter $(300 \mathrm{~Hz})$, before spike detection -see supplementary for details.

659 Images and graphs were processed and generated using Image J (National Institutes of 
662 Recoverin and cone arrestin quantification of ICC was performed using cell profiler 3.1.9. Total

663 graft area was quantified using Zen Blue image analysis wizard, and then each individual GFP ${ }^{+}$

664 cell cluster with associated area was categorized manually as ONL contact - cluster made

665 contact with the ONL but mostly remained in the subretinal space, partially incorporated -

666 the cluster was in line with the host ONL, however gaps or rosette like structures from

667 remaining host ONL reside below the graft area, or fully incorporated - the cluster replaced

668 sections of host ONL without gaps or rosettes from the host.

669

670 Panther was used for gene enrichment analysis (62). Differentially expressed genes from our

671 data set were run through the statistical overrepresentation test function using the whole

672 human genome as reference list. Fisher's exact test with calculated false discovery rate was

673 selected and output was condensed by hierarchical clustering of GO-terms to reduce

674 repetitive pathway findings. Morpheus (https://software.broadinstitute.org/morpheus) was

675 used to create heat maps.

676

677 Statistical analyses

678 Statistical significance was calculated using a one-way ANOVA with Tukeys multiple

679 comparison tests. Statistical significance is represented in the figures as follows: ${ }^{*}, p<.05$;

$680 * *, p<.01 ; * * *, p<.001 ; * * * *, p<.0005$, n.s.: not-statistically significant. Detailed statistical

681 analysis of transcriptional data and spike sorting see respective sections.

682

\section{Study approval}

684 All animal experiments were approved by the ethics committee of the TU Dresden and the 
bioRxiv preprint doi: https://doi.org/10.1101/2021.08.26.457641; this version posted August 28,2021 . The copyright holder for this preprint

(which was not certified by peer review) is the author/funder, who has granted bioRxiv a license to display the preprint in perpetuity. It is made available under aCC-BY-NC-ND 4.0 International license.

686 from European Union, German laws (Tierschutzgesetz), the ARVO statement for the Use of

687 Animals in Ophthalmic and Vision Research and the NIH Guide for the care and use of

688 laboratory work were strictly followed for all animal work.

689 
691

692 1. WHO. World report on vision [Internet]2019; https://www.who.int/publications-detail-

693 redirect/9789241516570. cited April 27, 2021

694 2. Mead B et al. Stem cell treatment of degenerative eye disease. Stem Cell Res.

$695 \quad 2015 ; 14(3): 243-257$.

696 3. Kolb H. Facts and Figures Concerning the Human Retina [Internet]. In: Kolb H, Fernandez

697 E, Nelson R eds. Webvision: The Organization of the Retina and Visual System. Salt Lake City

698 (UT): University of Utah Health Sciences Center; 1995:

699 4. Welby E et al. Isolation and Comparative Transcriptome Analysis of Human Fetal and iPSC-

700 Derived Cone Photoreceptor Cells. Stem Cell Rep. 2017;9(6):1898-1915.

701 5. Iraha S et al. Establishment of Immunodeficient Retinal Degeneration Model Mice and

702 Functional Maturation of Human ESC-Derived Retinal Sheets after Transplantation. Stem Cell

703 Rep. 2018;10(3):1059-1074.

704 6. Lin B, McLelland BT, Mathur A, Aramant RB, Seiler MJ. Sheets of human retinal progenitor

705 transplants improve vision in rats with severe retinal degeneration. Exp. Eye Res.

$706 \quad 2018 ; 174: 13-28$.

707 7. Mandai M et al. iPSC-Derived Retina Transplants Improve Vision in rd1 End-Stage Retinal-

708 Degeneration Mice. Stem Cell Rep. 2017;8(1):69-83. 
8. McLelland BT et al. Transplanted hESC-Derived Retina Organoid Sheets Differentiate,

710 Integrate, and Improve Visual Function in Retinal Degenerate Rats. Invest. Ophthalmol. Vis.

711 Sci. 2018;59(6):2586-2603.

9. Tu H-Y et al. Medium- to long-term survival and functional examination of human iPSC-

713 derived retinas in rat and primate models of retinal degeneration. EBioMedicine

$714 \quad 2018 ; 39: 562-574$.

10. Ribeiro J et al. Restoration of visual function in advanced disease after transplantation of

716 purified human pluripotent stem cell-derived cone photoreceptors [Internet]. Cell Rep.

717 2021;35(3). doi:10.1016/j.celrep.2021.109022

718

719

720

721

722

723

724 525.

727 15. Kim S et al. Generation, transcriptome profiling, and functional validation of cone-rich 728 human retinal organoids. Proc. Natl. Acad. Sci. 2019;116(22):10824-10833.

11. Collin J et al. CRX Expression in Pluripotent Stem Cell-Derived Photoreceptors Marks a

Transplantable Subpopulation of Early Cones. Stem Cells 2019;37(5):609-622.

12. Zerti D et al. Transplanted pluripotent stem cell-derived photoreceptor precursors elicit

conventional and unusual light responses in mice with advanced retinal degeneration. Stem Cells 2021;39(7):882-896.

13. Pfeiffer RL, Marc RE, Jones BW. Persistent remodeling and neurodegeneration in latestage retinal degeneration. Prog. Retin. Eye Res. 2020;74:100771.

14. Chang B et al. Retinal degeneration mutants in the mouse. Vision Res. 2002;42(4):517- 
731 Organoid. Stem Cell Rep. 2016;6(5):743-756.

734 Ther. [published online ahead of print: March 23, 2021]; doi:10.1089/hum.2020.321

736 cytoplasmic exchange. Nat. Commun. 2016;7:13028.

740 photoreceptors by a mechanism of cytoplasmic fusion. Nat. Commun. 2016;7:13537.

741 21. Gonzalez-Cordero A et al. Recapitulation of Human Retinal Development from Human

742 Pluripotent Stem Cells Generates Transplantable Populations of Cone Photoreceptors. Stem 743 Cell Rep. 2017;9(3):820-837.

744 22. Reichenbach A, Bringmann A. New functions of Müller cells. Glia 2013;61(5):651-678.

745 23. Gagliardi G et al. Characterization and Transplantation of CD73-Positive Photoreceptors

746 Isolated from Human iPSC-Derived Retinal Organoids. Stem Cell Rep. 2018;11(3):665-680.

747 24. Tikidji-Hamburyan A et al. Rods progressively escape saturation to drive visual responses 748 in daylight conditions. Nat. Commun. 2017;8(1):1813. 
25. Collin J et al. Using Zinc Finger Nuclease Technology to Generate CRX-Reporter Human

751 Precursors During Pluripotent Stem Cell Differentiation. Stem Cells Dayt. Ohio

$752 \quad 2016 ; 34(2): 311-321$.

26. Kaewkhaw R et al. Transcriptome Dynamics of Developing Photoreceptors in Three-

754 Dimensional Retina Cultures Recapitulates Temporal Sequence of Human Cone and Rod

755 Differentiation Revealing Cell Surface Markers and Gene Networks. STEM CELLS

$7562015 ; 33(12): 3504-3518$.

Technol. 2020;9(3):21.

29. Zweifel SA et al. Outer retinal tubulation: a novel optical coherence tomography finding.

Arch. Ophthalmol. 2009;127(12):1596-1602. 
771 primate models of retinal degeneration. Proc. Natl. Acad. Sci. 2016;113(1):E81-E90.

772 33. Ingram NT, Fain GL, Sampath AP. Elevated energy requirement of cone photoreceptors.

773 Proc. Natl. Acad. Sci. 2020;117(32):19599-19603.

774

775

34. Kljavin IJ, Reh TA. Muller cells are a preferred substrate for in vitro neurite extension by rod photoreceptor cells. J. Neurosci. 1991;11(10):2985-2994.

35. Tsai ELS et al. Modeling of Photoreceptor Donor-Host Interaction Following

Transplantation Reveals a Role for Crx, Müller Glia, and Rho/ROCK Signaling in Neurite

Outgrowth. Stem Cells 2019;37(4):529-541.

36. Wang X, lannaccone A, Jablonski MM. Contribution of Müller cells toward the regulation of photoreceptor outer segment assembly. Neuron Glia Biol. 2005;1:1-6.

37. Cowan CS et al. Cell Types of the Human Retina and Its Organoids at Single-Cell Resolution. Cell 2020;182(6):1623-1640.e34.

38. Mellough CB et al. IGF-1 Signaling Plays an Important Role in the Formation of ThreeDimensional Laminated Neural Retina and Other Ocular Structures From Human Embryonic Stem Cells. Stem Cells 2015;33(8):2416-2430.

39. Reichman S et al. Generation of Storable Retinal Organoids and Retinal Pigmented Epithelium from Adherent Human iPS Cells in Xeno-Free and Feeder-Free Conditions. Stem Cells 2017;35(5):1176-1188.

40. Zhong $X$ et al. Generation of three-dimensional retinal tissue with functional photoreceptors from human iPSCs. Nat. Commun. 2014;5:4047. 
792 from Human Pluripotent Stem Cells. Sci. Rep. 2017;7(1):766.

42. Garita-Hernandez $M$ et al. Restoration of visual function by transplantation of

43. Chavez A et al. Highly efficient Cas9-mediated transcriptional programming. Nat.

Methods 2015;12(4):326-328.

797

798

44. Gibson DG et al. Creation of a Bacterial Cell Controlled by a Chemically Synthesized

Genome. Science 2010;329(5987):52-56.

45. Busskamp V et al. Genetic reactivation of cone photoreceptors restores visual responses in retinitis pigmentosa. Science 2010;329(5990):413-417.

46. Busskamp V et al. Rapid neurogenesis through transcriptional activation in human stem cells. Mol. Syst. Biol. 2014;10(11):760.

\section{Lee J-H et al. A Robust Approach to Identifying Tissue-Specific Gene Expression}

Regulatory Variants Using Personalized Human Induced Pluripotent Stem Cells. PLOS Genet. 2009;5(11):e1000718.

48. Eberle D, Santos-Ferreira T, Grahl S, Ader M. Subretinal transplantation of MACS purified photoreceptor precursor cells into the adult mouse retina. J. Vis. Exp. JoVE 2014;(84):e50932.

49. Völkner M et al. Mouse Retinal Organoid Growth and Maintenance in Longer-Term Culture. Front. Cell Dev. Biol. 2021;9:645704. 
$8132019 ; 1834: 119-141$.

814 51. Fabig $\mathrm{G}$ et al. Labeling of ultrathin resin sections for correlative light and electron

815 microscopy. Methods Cell Biol. 2012;111:75-93.

816 52. Eberle D et al. Outer segment formation of transplanted photoreceptor precursor cells.

$817 \quad$ PloS One 2012;7(9):e46305.

818 53. Picelli S et al. Smart-seq2 for sensitive full-length transcriptome profiling in single cells.

819 Nat. Methods 2013;10(11):1096-1098.

820 54. Zentgraf J, Rahmann S. Fast lightweight accurate xenograft sorting. Algorithms Mol. Biol.

$821 \quad A M B 2021 ; 16(1): 2$.

822 55. Wu TD, Nacu S. Fast and SNP-tolerant detection of complex variants and splicing in short

823 reads. Bioinformatics 2010;26(7):873-881.

824 56. Liao Y, Smyth GK, Shi W. featureCounts: an efficient general purpose program for

825 assigning sequence reads to genomic features. Bioinforma. Oxf. Engl. 2014;30(7):923-930.

826 57. Love MI, Huber W, Anders S. Moderated estimation of fold change and dispersion for

827 RNA-seq data with DESeq2. Genome Biol. 2014;15(12):550.

828 58. Ignatiadis N, Huber W. Covariate powered cross-weighted multiple testing [Internet].

829 ArXiv170105179 Stat [published online ahead of print: June 21,

830 2021];http://arxiv.org/abs/1701.05179. cited July 12, 2021 
831 59. Ignatiadis N, Klaus B, Zaugg JB, Huber W. Data-driven hypothesis weighting increases

832 detection power in genome-scale multiple testing. Nat. Methods 2016;13(7):577-580.

833 60. Reh M, Lee M-J, Schmierer J, Zeck G. Spatial and temporal resolution of optogenetically

834 recovered vision in ChR2-transduced mouse retina. J. Neural Eng. [published online ahead of

835 print: February 5, 2021]; doi:10.1088/1741-2552/abe39a

836 61. Szécsi L, Kacsó Á, Zeck G, Hantz P. Interactive Light Stimulus Generation with High

837 Performance Real-Time Image Processing and Simple Scripting. Front. Neuroinformatics

$838 \quad 2017 ; 11: 70$.

839 62. Mi H et al. PANTHER version 16: a revised family classification, tree-based classification

840 tool, enhancer regions and extensive API. Nucleic Acids Res. 2021;49(D1):D394-D403. 


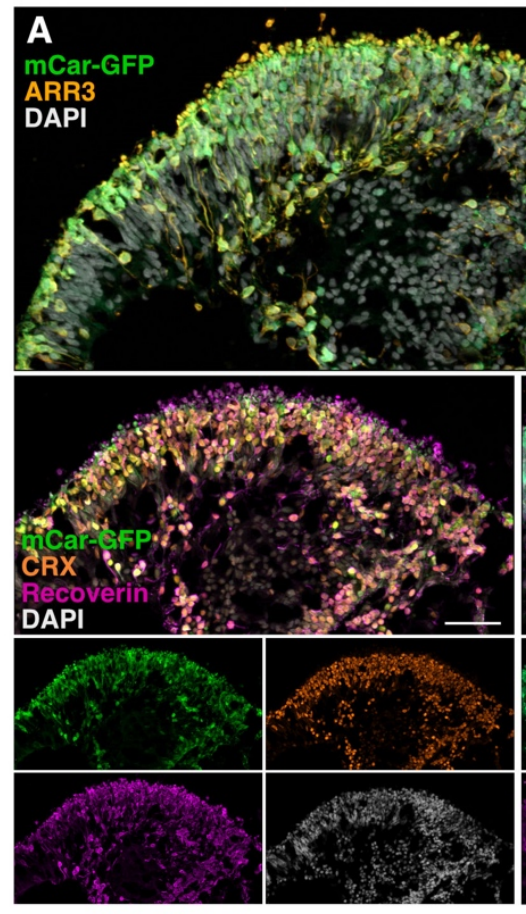

C
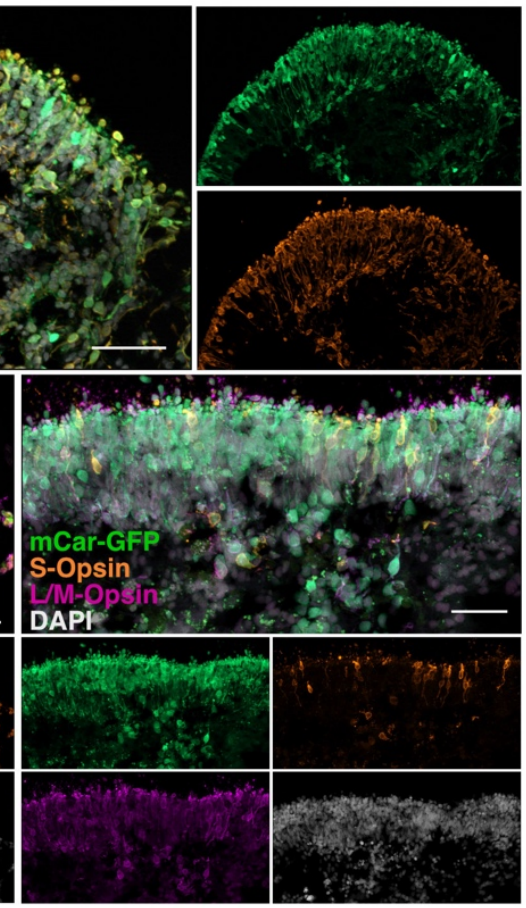

Day 270 vs Day 200 cones cellular compartment
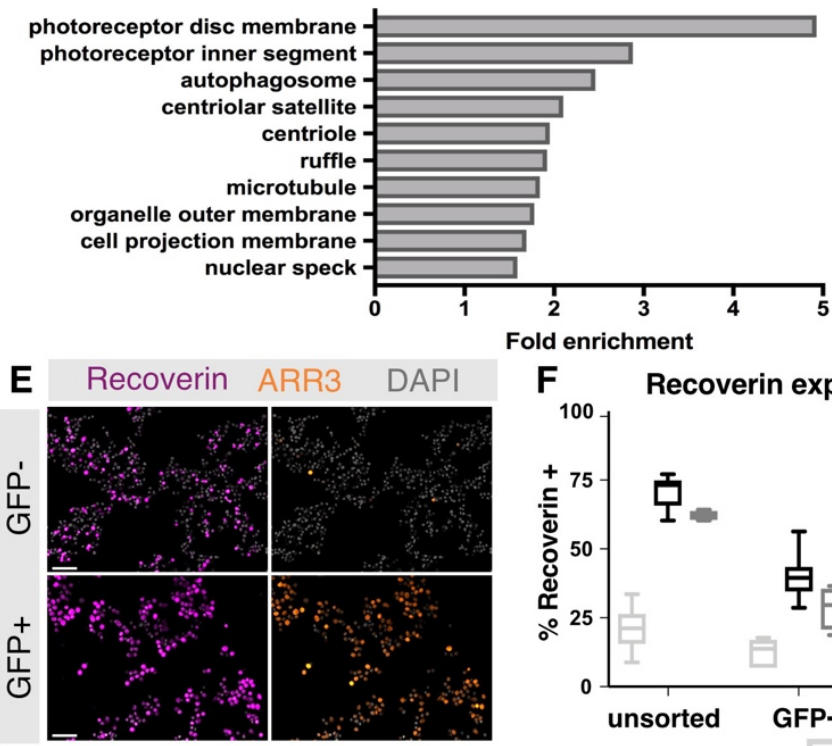

$\mathbf{F}$

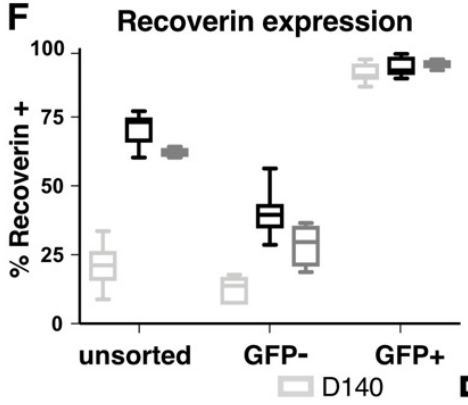

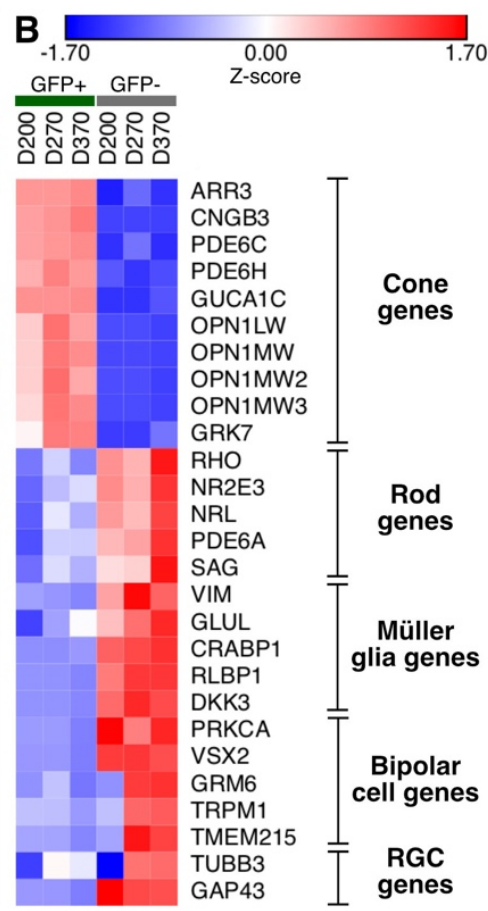

GFP+ cells (cones) with
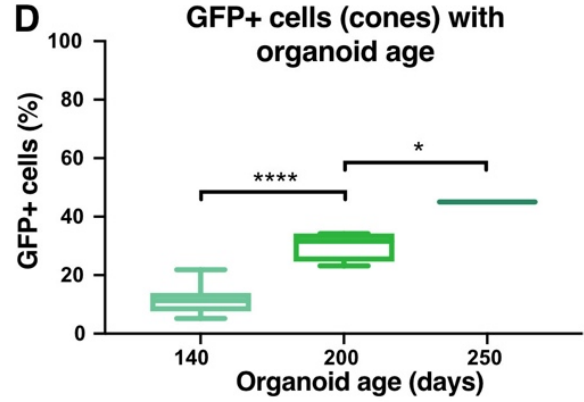

G Cone arrestin expression

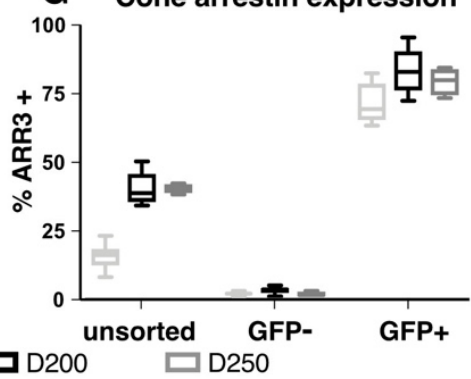

Figure 1: Generation and characterisation of a cone specific reporter line - D240 mCar-GFP derived retinal organoid cryosections show (A) co-staining of mCar-driven GFP with cone (ARR3, S-Opsin, L/M-opsin) and photoreceptor specific (CRX, recoverin) proteins. (B) Heat map of z-scores in major retinal cell type marker gene expression in GFP+ and GFP- cells sorted from mCar-GFP reporter organoids at D200, D270 and D370 post differentiation. (C) Gene ontology term cellular compartment over-representation analysis of D270 GFP+ cells compared with D200 GFP+ cells. (D) Proportion of GFP+ cells with organoid age. (E) Immunocytochemistry of GFP, recoverin and ARR3 expression in GFP+ and GFP- FAC-sorted fractions and quantification of immunocytochemical staining of (F) recoverin and (G) cone arrestin in unsorted, GFP+ and GFP-sorted fractions. 
bioRxiv preprint doi: https://doi.org/10.1101/2021.08.26.457641; this version posted August 28, 2021. The copyright holder for this preprint (which was not certified by peer review) is the author/funder, who has granted bioRxiv a license to display the preprint in perpetuity. It is made available under aCC-BY-NC-ND 4.0 International license.

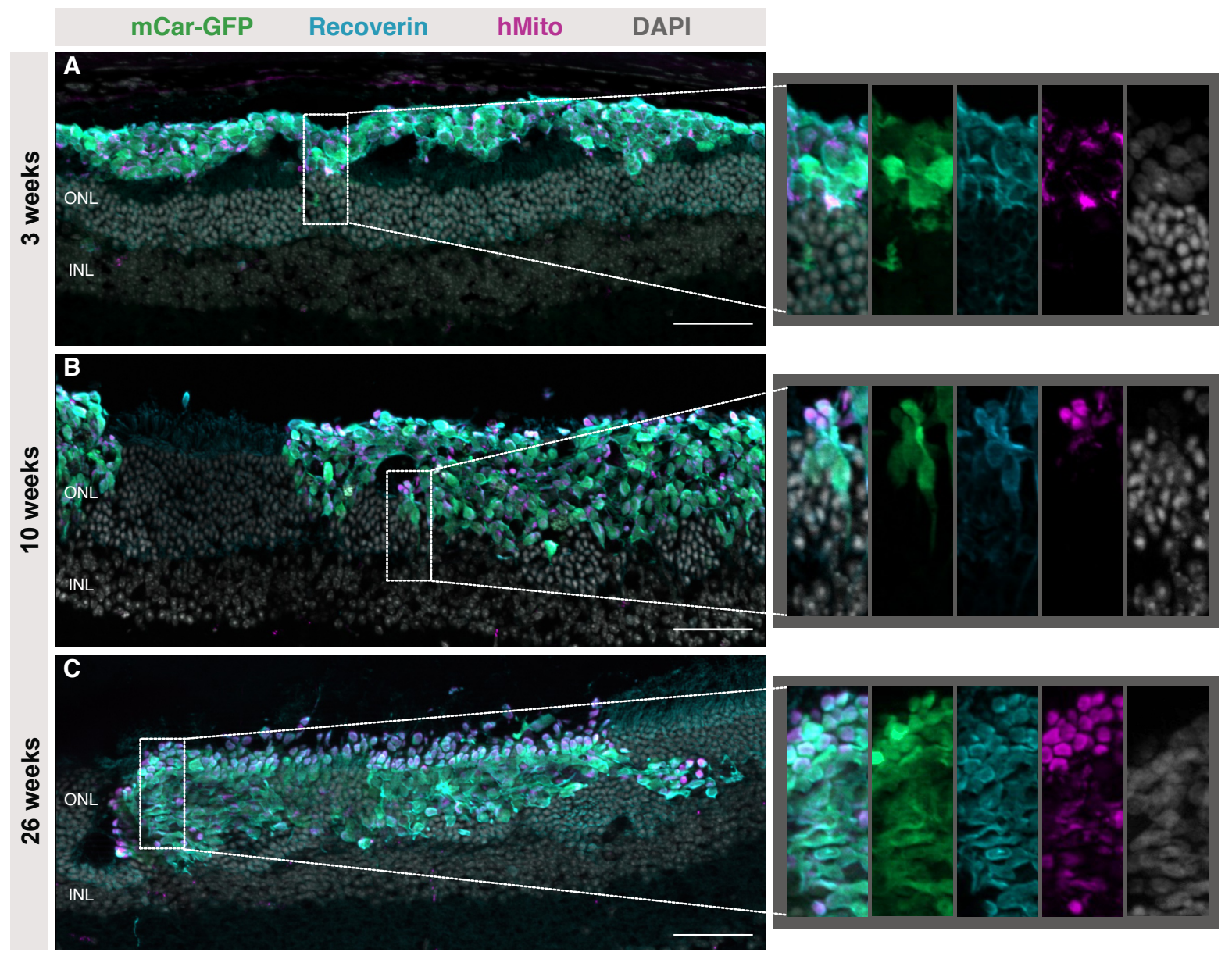

Figure 2: Extensive incorporation of transplanted cones into the Cpfl1 host retina with increased time since transplantation - Cryosections of retina transplanted with $\mathrm{mCar}^{-G_{F P}+}$ cells at $\mathrm{D} 200$ post-differentiation stained with GFP, recoverin, human mitochondria and DAPI show (A) minimal donor-host interaction 3 weeks posttransplantation and (B) large cell clusters incorporated into the host retina at 10 weeks post-transplantation, with areas of round mitochondria rich outgrowths towards the RPE and axon like extensions projected towards the inner nuclear layer (see zoomed area). (C) By 26 weeks, grafts displayed even more abundant mitochondria rich outgrowths (see zoomed area). Scale bars in all immunohistochemical images $50 \mu \mathrm{m}$. RPE: retinal pigment epithelium, DAPI: 4',6-diamidino-2-phenylindole 

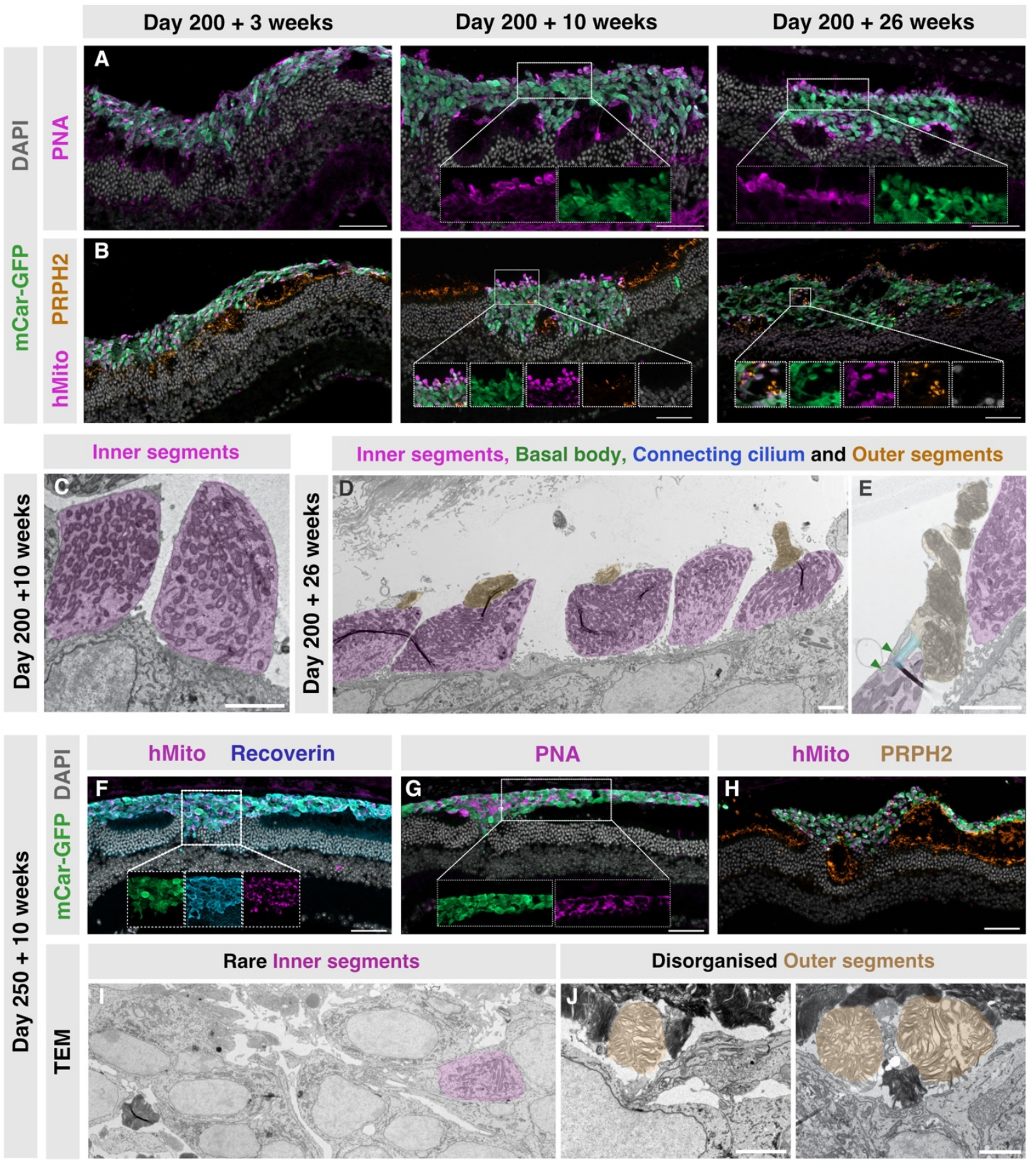

Figure 3: Graft development, polarisation and inner and outer segment formation - Cryosections of retina transplanted with D200 mCar-GFP' ${ }^{+}$cells were stained with (A) PNA showing more localised PNA binding with longer transplantations times. (B) PRPH2 shows most abundant staining at 26 weeks post-transplantation. TEM of ultrathin sections of eyes transplanted with D200 cones revealed (C) inner segments (purple) at 10 weeks post transplantation, (D) inner (purple) and outer segments (orange) and (E) occasionally basal bodies (green arrows) and connecting cilium (blue overlay) at 26 weeks post-transplantation. Cryosections of retina transplanted with mCar-GFP' cells at day 250 post-differentiation showed (F) minimal donor-host interaction and few mitochondria rich outgrowths, $(G)$ dispersed PNA binding and $(H)$ little PRPH2 staining. TEM of the D250 transplanted cones showed (I) few inner segments and (J) occasional disorganised outer segments. Scale bars in all Basal body, CC: Connecting cilium, TEM: Transmission electron microscopy, PNA: Peanut agglutinin, PRPH2; Peripherin2 
bioRxiv preprint doi: https://doi.org/10.1101/2021.08.26.457641; this version posted August 28, 2021. The copyright holder for this preprint (which was not certified by peer review) is the author/funder, who has granted bioRxiv a license to display the preprint in perpetuity. It is made available under aCC-BY-NC-ND 4.0 International license.

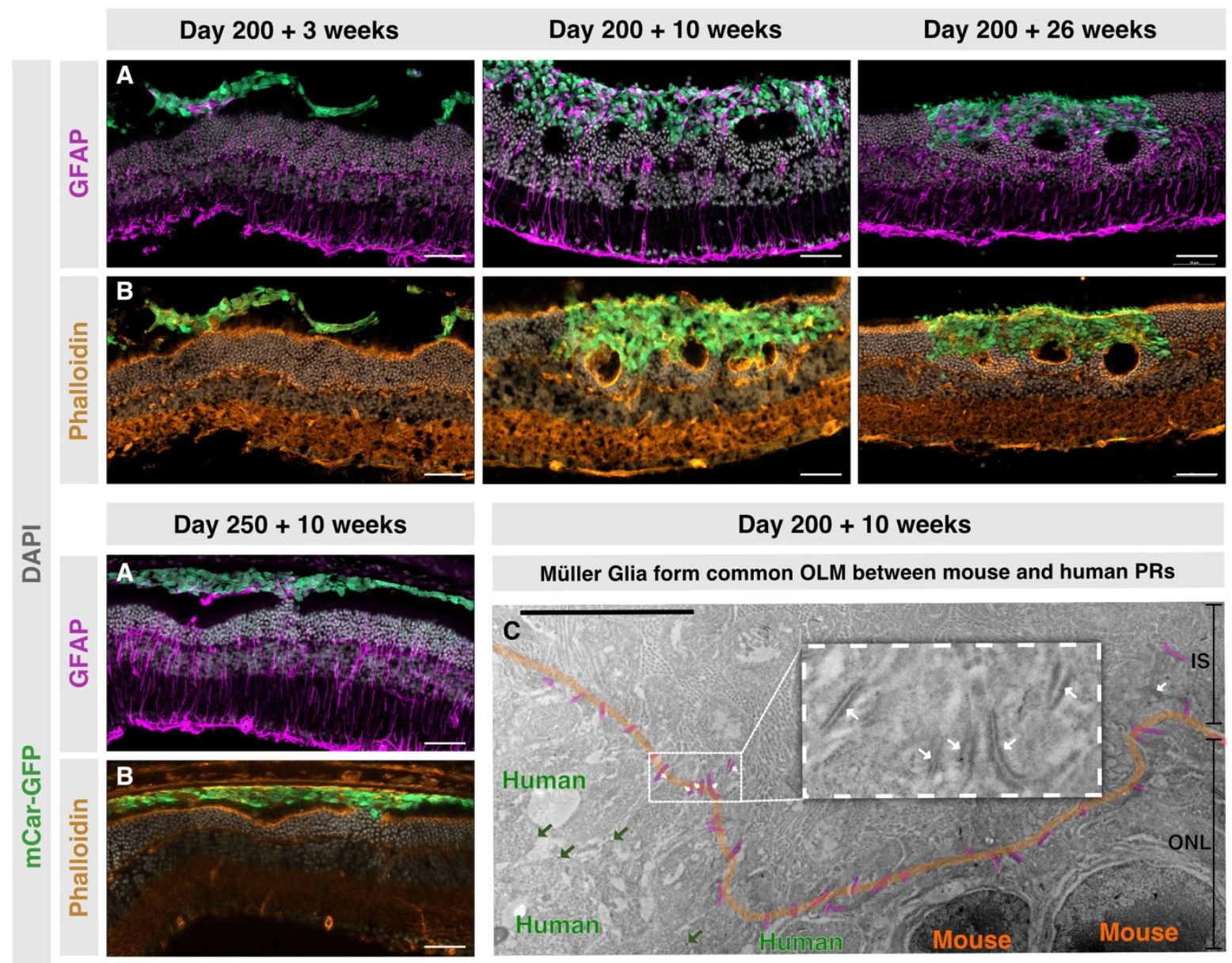

Day $200+26$ weeks

Figure 4: Host müller glia interaction with human cone grafts - Cryosections of retina transplanted with mCar-

GFP+ cells at D200 or D250 post-differentiation showed (A) Müller glia beginning to extend processes into areas where the graft contacted host ONL (D200+3 weeks, D250+10 weeks), and extensively intermingling with grafts (D200+10 and D200+26 weeks) which had incorporated into the host ONL. (B) Phalloidin staining indicates that a common continuous OLM forms when the human cones incorporate into the host ONL. (C) Immunogold labelling confirms the formation of a common OLM between mouse and human photoreceptors. Dark green arrows indicate examples of immunogold 10nm labelling of human ARR3, thick orange line indicates the position of the OLM, yellow strokes indicate adherens junctions between mouse Müller glia and both mouse and human photoreceptors. (D) 3D reconstruction of GFAP positive Müller glia processes extending around human cones. Scale bars in all immunohistochemical images $50 \mu \mathrm{m}$, for CLEM images $6 \mu \mathrm{m}$ and in 3D reconstruction grid lines are $50 \mu \mathrm{m}$. OLM: outer limiting membrane 
bioRxiv preprint doi: https://doi.org/10.1101/2021.08.26.457641; this version posted August 28,2021 . The copyright holder for this preprint (which was not certified by peer review) is the author/funder, who has granted bioRxiv a license to display the preprint in perpetuity. It is made available under aCC-BY-NC-ND 4.0 International license.

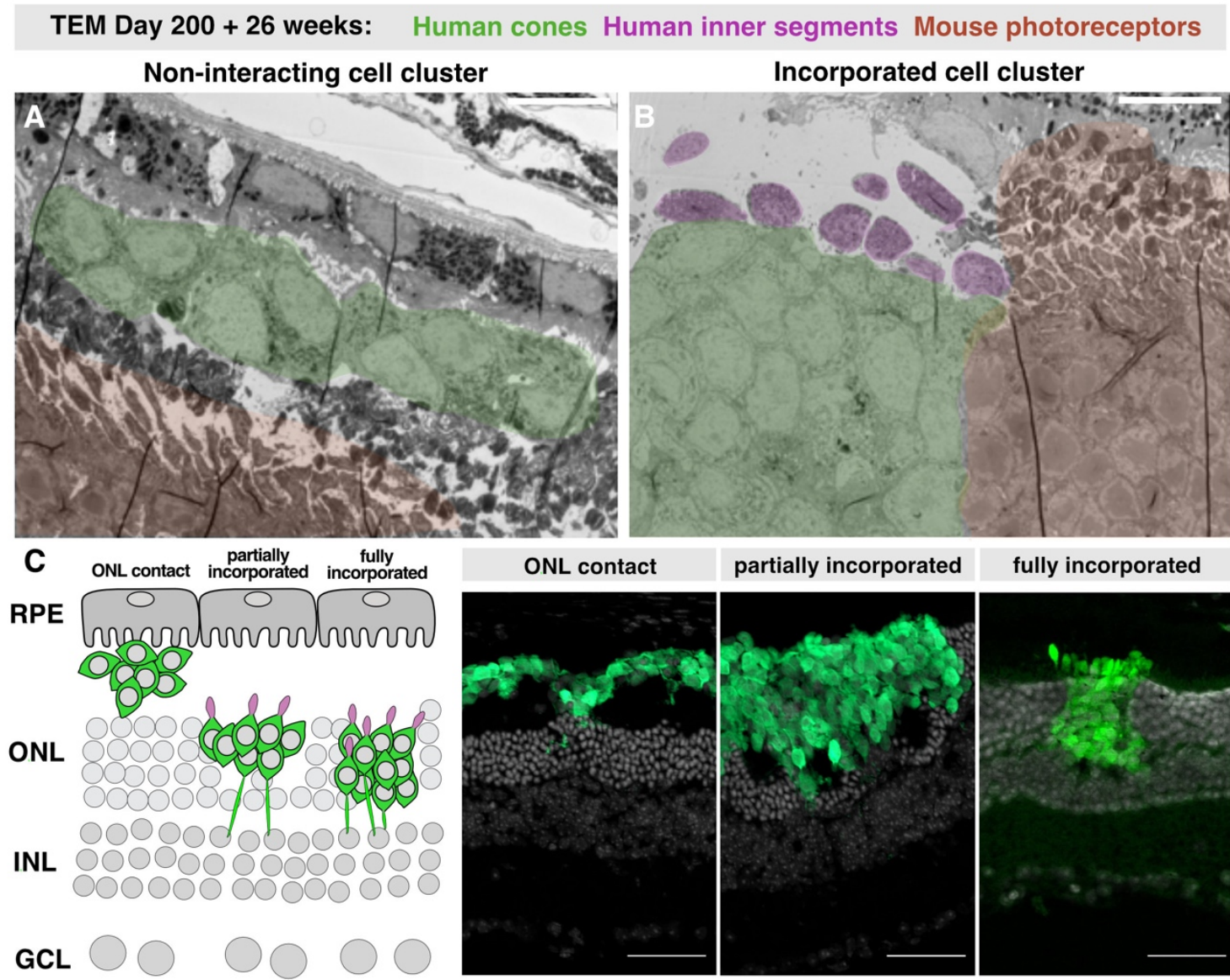

D
Partially incorporated Fully incorporated

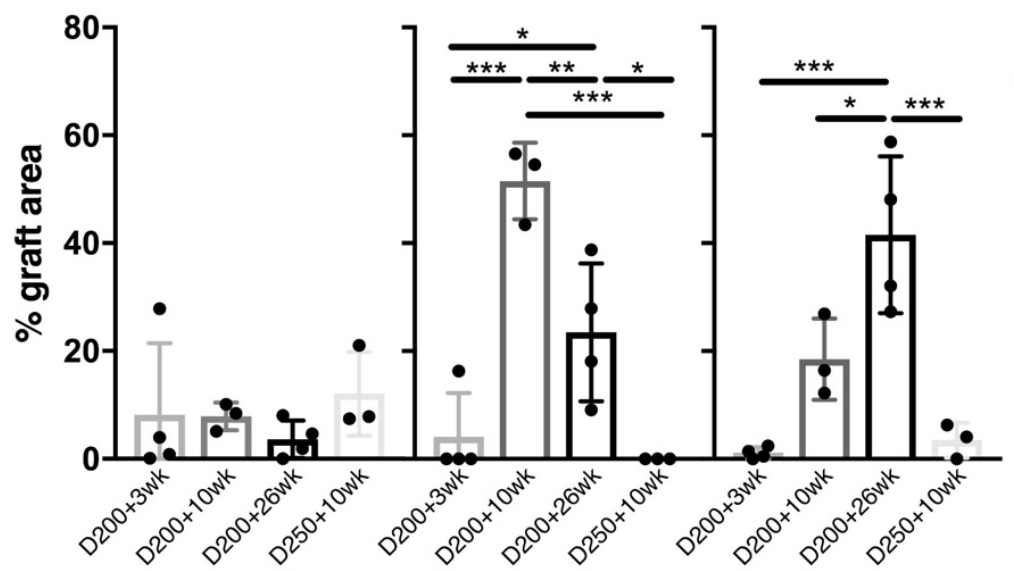

E

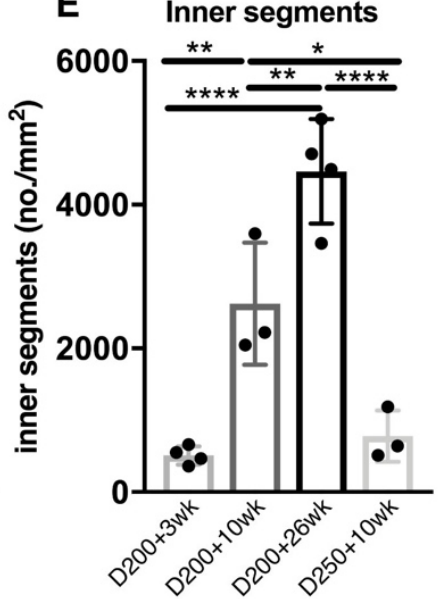

Figure 5: Interactive grafts more readily develop inner segments - Representative TEM of ultrathin retinal sections where some cone clusters (green overlay) within the same mouse eye (A) remain in the subretinal space or (B) incorporate into the host ONL (mouse photoreceptors orange overlay) and develop inner segments (purple overlay). (C) Schematic representation and example retinal cryosections for the classification of donor-host interaction into ONL contact, partially or fully incorporated. (D) Quantification of retinal cluster interaction with the host retina by area ( $n=3-4$ eyes). (E) Number of mitochondria rich presumed IS at each timepoint $(n=3-4$ eyes). Scale bars in all immunohistochemical images $50 \mu \mathrm{m}$ and for all TEM images $10 \mu \mathrm{m}$. IS: Inner segment, ONL: Outer nuclear layer, TEM: Transmission electron microscopy. Data displayed as mean and SD $* p<0.05$, $* * \mathrm{p} \leq 0.01, * * * \mathrm{p} \leq 0.001, * * * * \mathrm{p} \leq 0.0001$ 
A

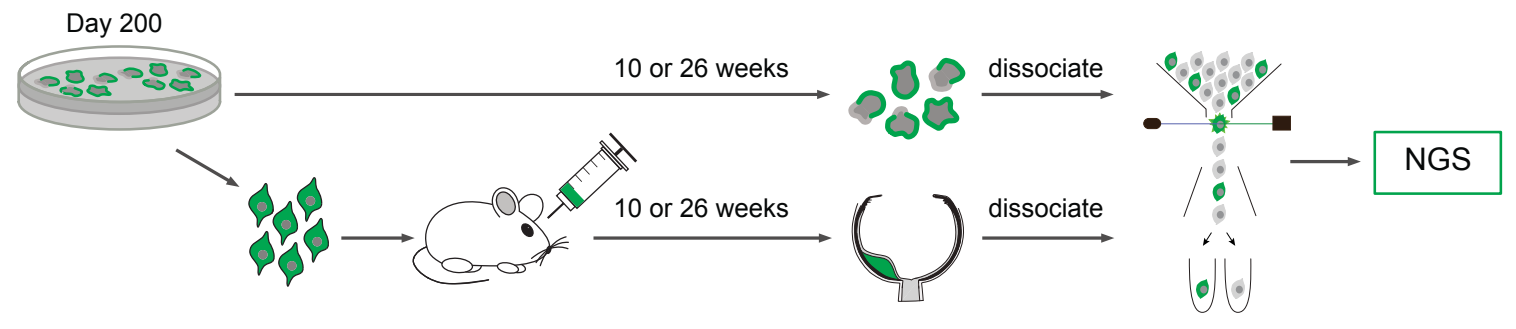

B

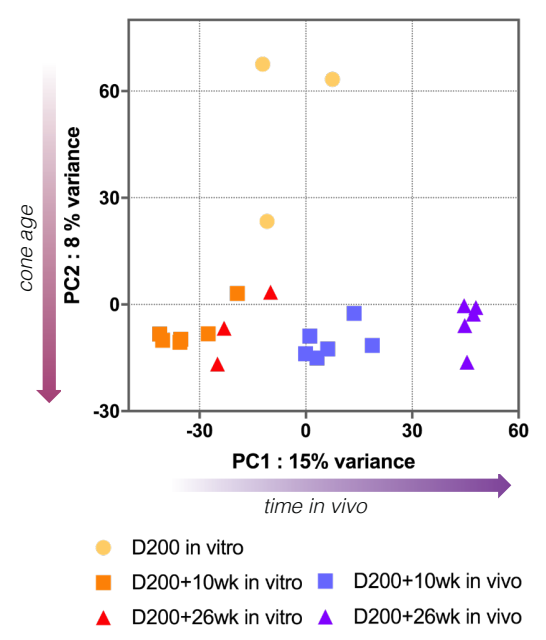

D

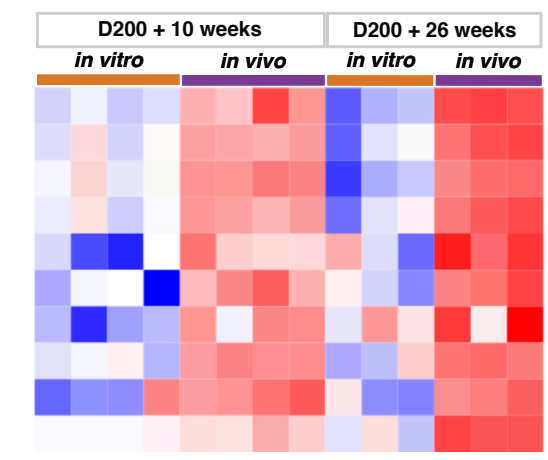

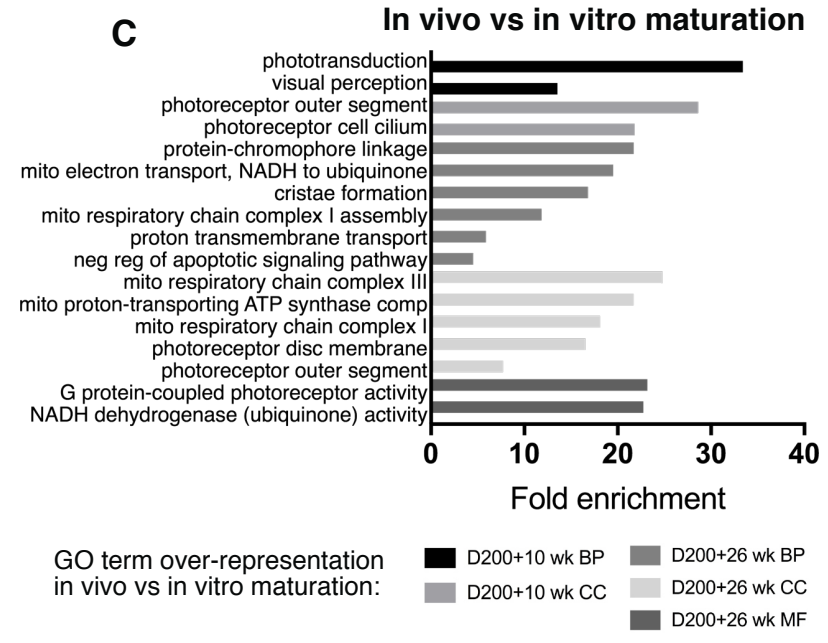

E

Mitochondrial complex 1

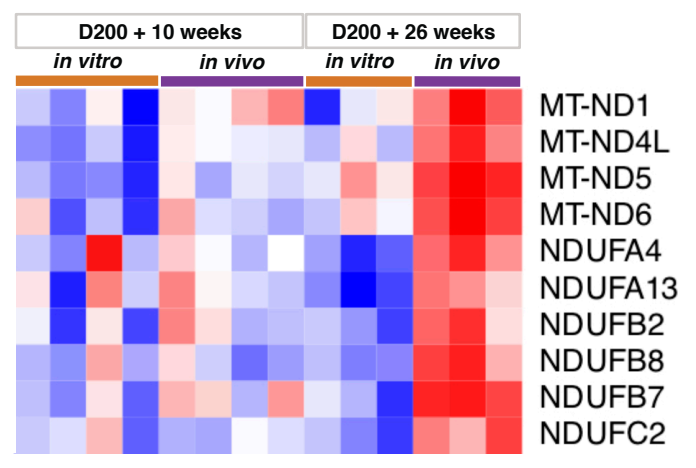

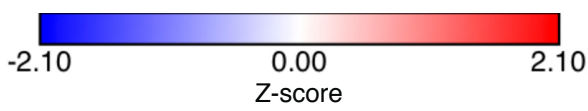

Figure 6: Transcriptional profiling of transplanted cones compared to age-matched organoid derived cones - (A) Schematic representation of $\mathrm{mCar}_{-G^{+}} \mathrm{GF}^{+}$cone sequencing work-flow. (B) principal component analysis of the top 500 differentially regulated genes. (C) GO term pathway over representation analysis of in vivo matured vs in vitro matured cones. Heat maps of z-scores for genes involved in (D) visual perception and (E) mitochondrial complex 1. 
bioRxiv preprint doi: https://doi.org/10.1101/2021.08.26.457641; this version posted August 28, 2021. The copyright holder for this preprint (which was not certified by peer review) is the author/funder, who has granted bioRxiv a license to display the preprint in perpetuity. It is made available under aCC-BY-NC-ND 4.0 International license.

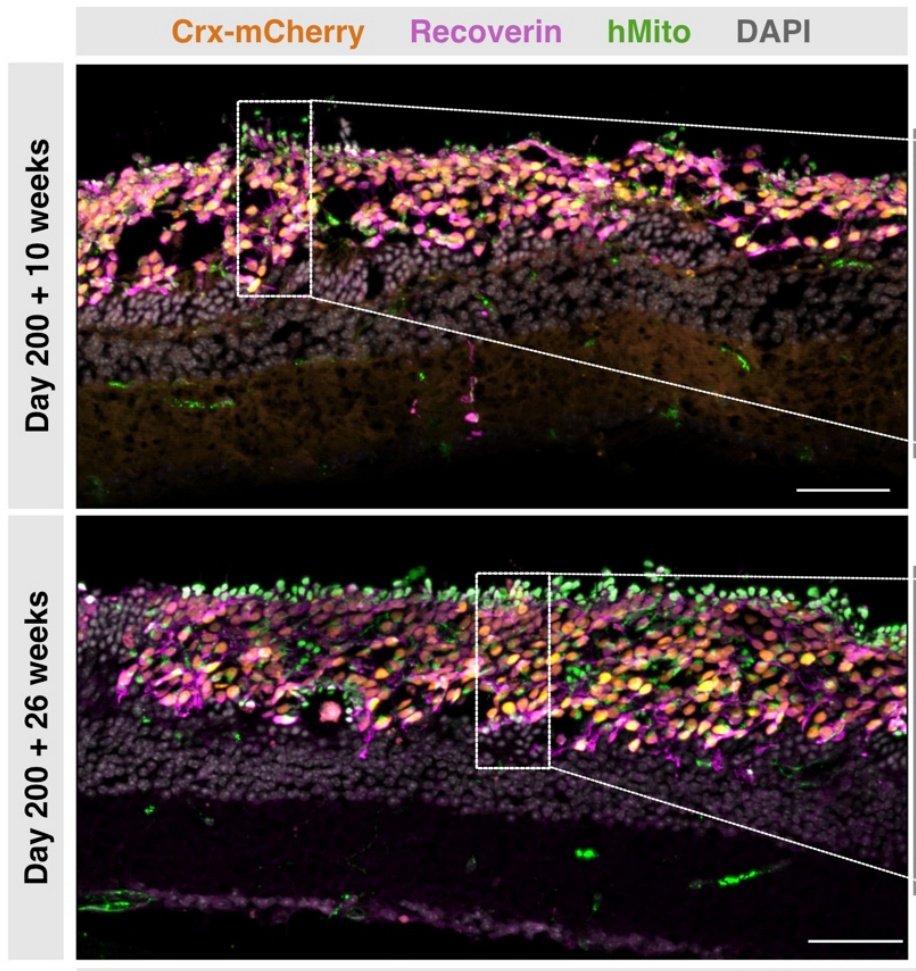

Crx-mCherry/ARR3
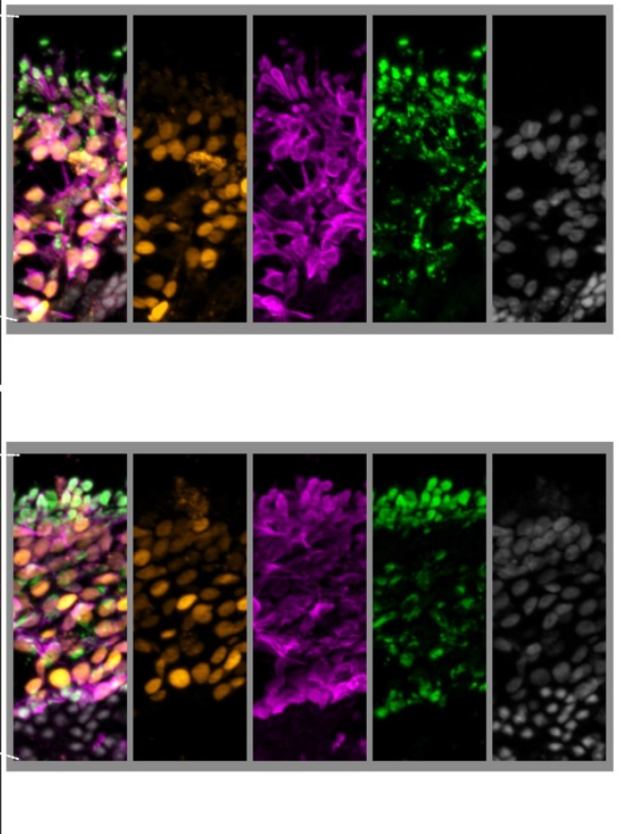

DAPI

PNA
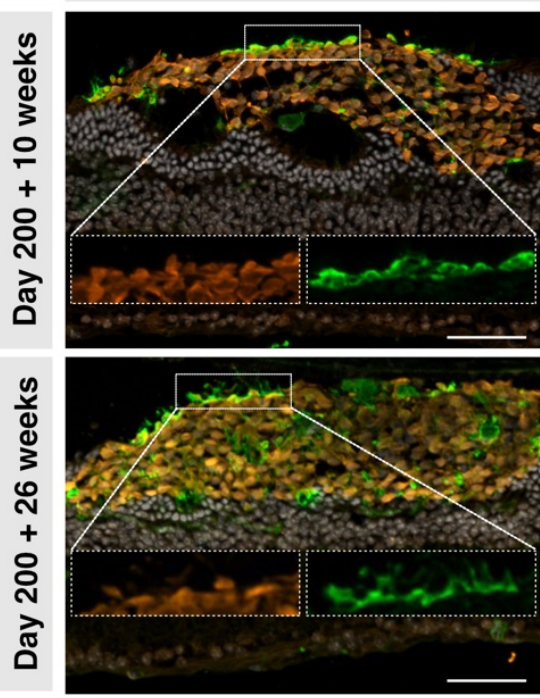

PRPH2 hMito
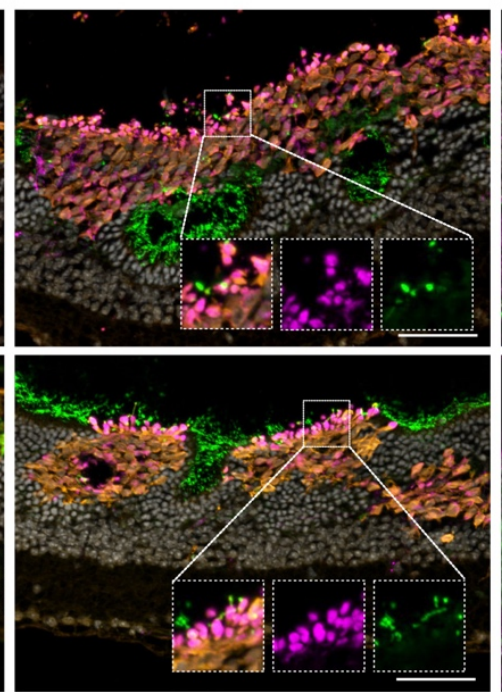

GFAP Phalloidin
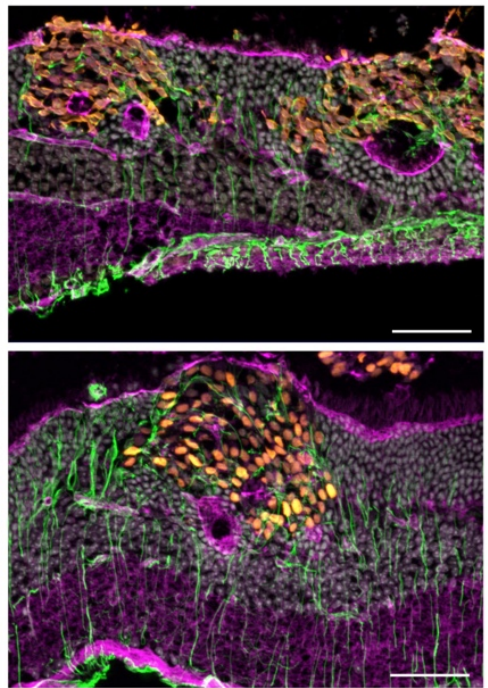

Figure 7: Crx-mCherry ${ }^{+}$grafts also display extensive incorporation and polarisation - Retinal cryosections of CrxmCherry ${ }^{+}$grafts transplanted at D200 stained with recoverin, human mitochondria and DAPI shows (A) by 10 weeks large cell clusters incorporate into the host retina with areas of round mitochondria rich outgrowths towards the RPE and axon like extensions projected towards the inner nuclear layer (see zoomed area). (B) By 26 weeks grafts displayed even more abundant mitochondria rich outgrowths (see zoomed area). (C) PNA is bound in a more localised fashion towards the RPE. (D) Peripherin- 2 is more extensively expressed at 26 weeks post transplantation and (D) Müller glia processes intermingle throughout the graft. Scale bars in all 


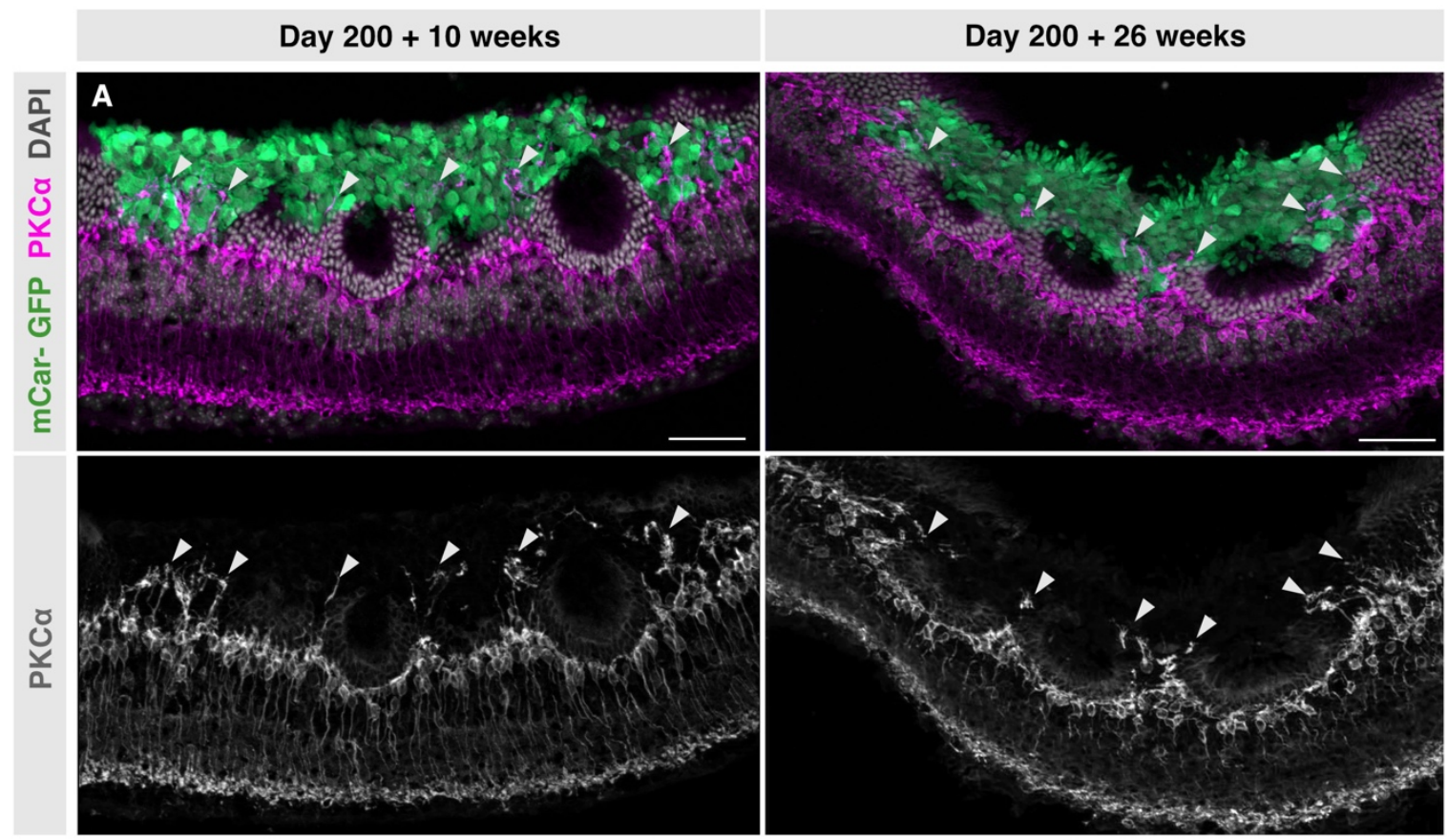

Synaptic proximity
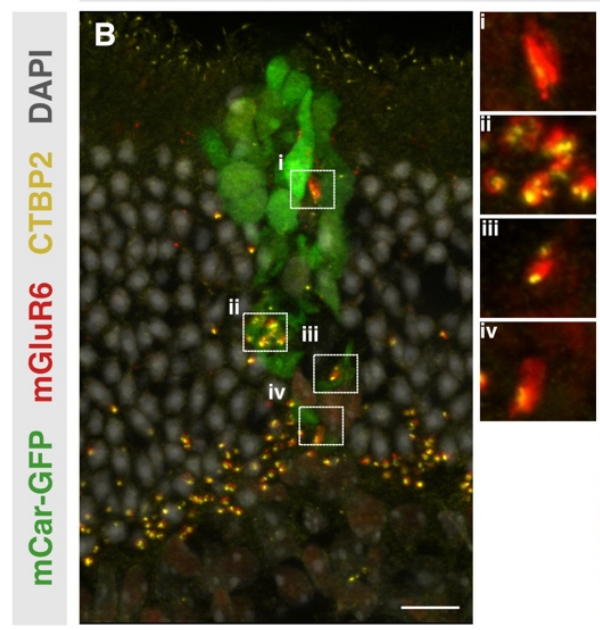

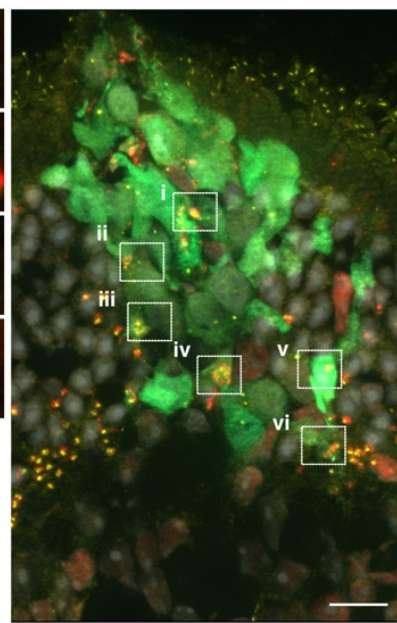

Ribbon synapses
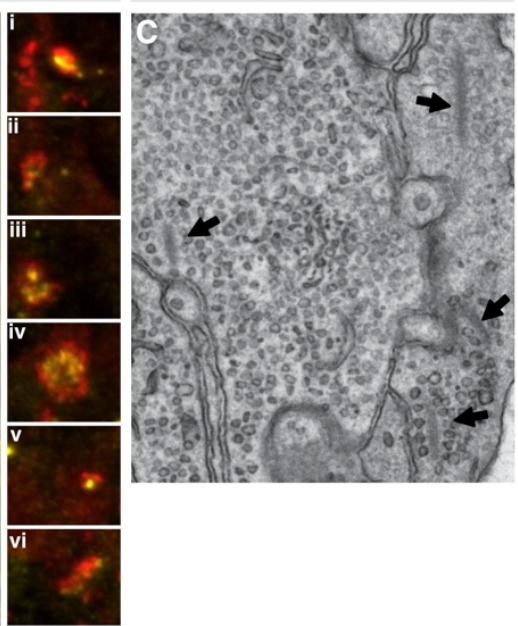

Figure 8: Putative synapse formation between transplanted human cones and host bipolar cells Immunolabelled cryosections of Cpfl1 retina transplanted with mCar-GFP ${ }^{+}$cells show (A) extensive dendrite extensions into the cone cell graft from PKCalpha ${ }^{+}$rod bipolar cells. White arrowheads indicate areas of dendrite extensions. (B) Close association of the presynaptic ribbon synapse marker CTBP2 and the bipolar postsynaptic marker mGluR6. (C) Representative ribbons and vesicles, components of the photoreceptor presynapse, highlighted by arrows in a TEM image of an incorporated graft. Scale bars in all immunohistochemical images 50 $\mu \mathrm{m}$ and TEM $500 \mathrm{~nm}$ 

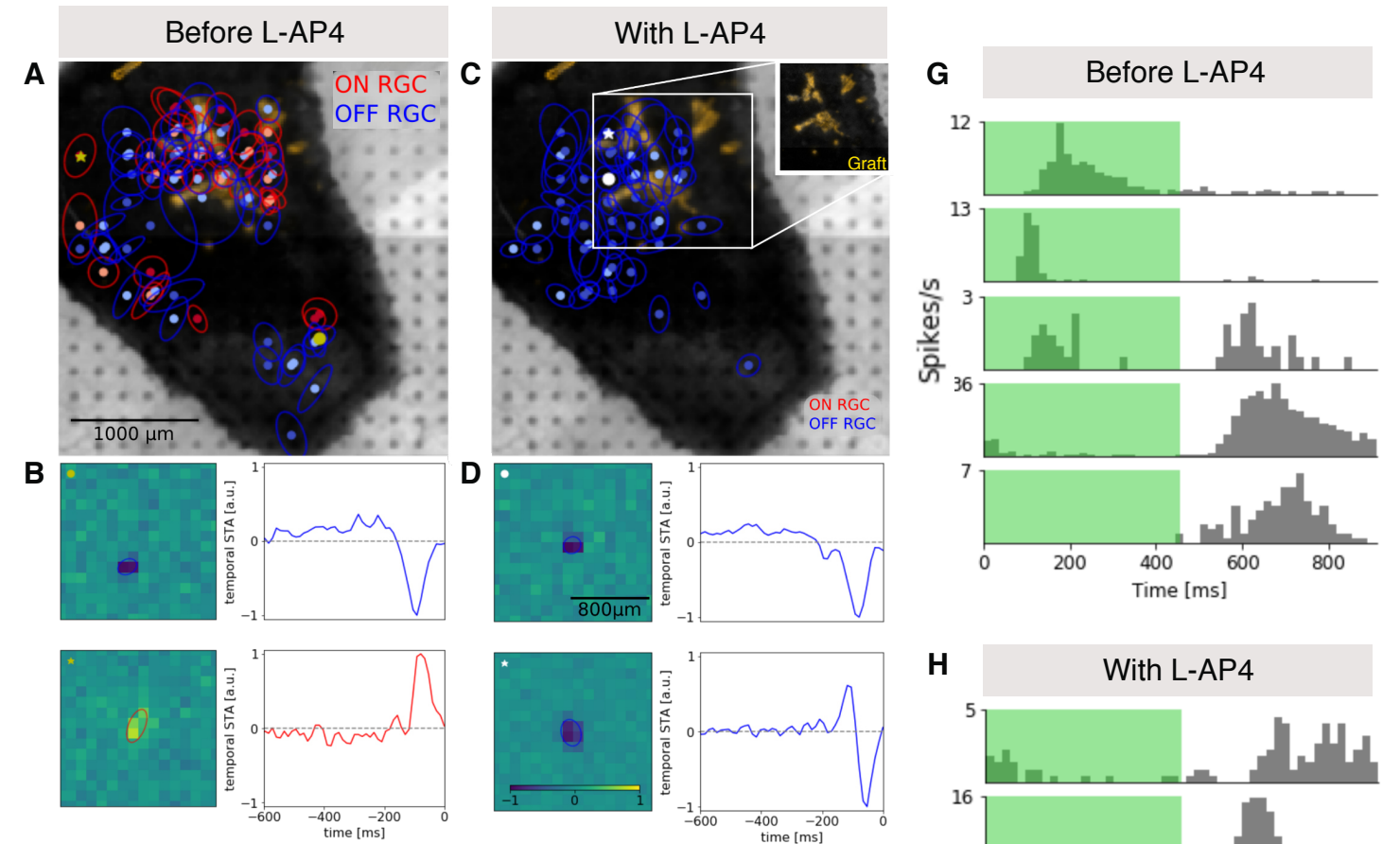

E

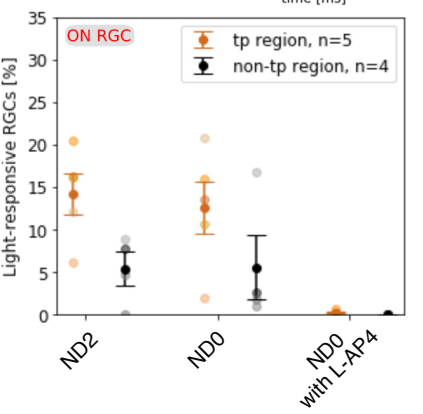

$\mathbf{F}$

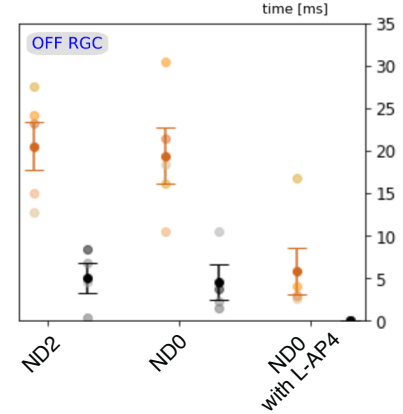

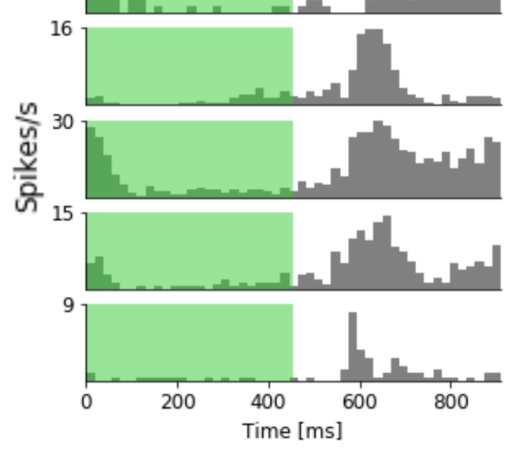

Figure 9: Increased RGC activity after Crx-mCherry ${ }^{+}$photoreceptor transplantation - (A) Receptive Fields for ON and OFF RGCs detected following photopic stimulation (NDO). (B) Exemplary receptive fields and temporal STA for the two cells labeled with $*$ and $\circ$ in (A). (C) Receptive Fields where only OFF RGCs remain following photopic stimulation after addition of L-AP4 (ND0 after L-AP4). (D) Exemplary receptive fields and temporal STA for the two cells labeled with $*$ and $\circ$ in (C). Percentage of light responsive (E) ON RGCs and (F) OFF RGCs detected under mesopic (ND2), photopic (ND0) and photopic stimulation with the addition of L-AP4 (NDO after L-AP4). (G-H) Response of 5 different RGCs during full-field photopic (NDO) ON-OFF flicker stimulation. The bin width is $20 \mathrm{~ms}$ and in total a number of 120 stimulus repetitions were performed. (G) Distinct ON, OFF and ON-OFF RGC responses to flicker stimulation before addition of L-AP4. (H) Distinct OFF RGC responses to flicker stimulation with addition of L-AP4 only remain in regions with transplant. RGC: Retinal ganglion cell, L-AP4: L-2-amino-4phosphonobutyric acid. 